\title{
Hyperchaos Numerical Simulation and Control in a 4D Hyperchaotic System
}

\author{
Junhai Ma and Yujing Yang \\ College of Management and Economics, Tianjin University, Tianjin 300072, China \\ Correspondence should be addressed to Junhai Ma; lzqsly@126.com
}

Received 15 May 2013; Revised 29 July 2013; Accepted 19 August 2013

Academic Editor: Oswaldo Luiz do Valle Costa

Copyright (c) 2013 J. Ma and Y. Yang. This is an open access article distributed under the Creative Commons Attribution License, which permits unrestricted use, distribution, and reproduction in any medium, provided the original work is properly cited.

\begin{abstract}
A hyperchaotic system is introduced, and the complex dynamical behaviors of such system are investigated by means of numerical simulations. The bifurcation diagrams, Lyapunov exponents, hyperchaotic attractors, the power spectrums, and time charts are mapped out through the theory analysis and dynamic simulations. The chaotic and hyper-chaotic attractors exist and alter over a wide range of parameters according to the variety of Lyapunov exponents and bifurcation diagrams. Furthermore, linear feedback controllers are designed for stabilizing the hyperchaos to the unstable equilibrium points; thus, we achieve the goal of a second control which is more useful in application.
\end{abstract}

\section{Introduction}

In recent years, hyperchaotic systems have been extensively studied because of exhibiting at least two positive Lyapunov exponents. That is to say, the dynamics of the system expand in more than one direction and generate a much more complex attractor compared with the chaotic system with only one positive Lyapunov exponent, which is studied by Rech et al. $[1,2]$. It means that hyperchaotic systems generate much more dynamical behaviors compared with chaotic systems [3-5]. Historically, the noted four-dimensional hyperchaotic system was firstly reported by Lörenz [6]. Chen et al. [7, 8] have studied the dynamics of the fractional-order generalizations of the Lörenz hyperchaos equation and found a new chaotic attractor in a simple three-dimensional autonomous system, which resembles some familiar features from both the Lörenz and Rössler attractors. Nowadays, the application of the hyperchaos is becoming a hot topic in the field of chaos. Wang $[9,10]$ has presented a multiscroll chaotic system generated from a new quadratic autonomous system and introduced a new three-dimensional quadratic autonomous system, which can generate a pair of double-wing chaotic attractors. Moreover, he has also found that the system can generate three-wing and four-wing chaotic attractors with very complicated topological structures over a large range of parameters. On the basis of the analysis above, several useful issues are investigated either analytically or numerically. Ma et al. [11-13] have expressed many of the dynamical systems generating both hyperchaotic and chaotic behaviors, such as quadratic dynamic behaviors, and analyzed it furthermore in various fields. Yang [14] has analyzed an input control of exponential synchronization for a four-dimensional chaotic system and obtained some valuable conclusions. Wang et al. [15] have studied an asynchronous communication system based on the hyperchaotic system of 6th-order cellular neural network and drew some useful conclusions. Correia and Rech [16] have introduced a state feedback control to the first equation based on Wang and proposed a method that also considers the magnitude of the second largest Lyapunov exponent to numerically characterize points with hyperchaotic behavior, in which two parameters are simultaneously varied, and they obtained some practical and theoretical significance in the practice. Besides this, Choudhury and Van Gorder [17] have discovered hyperchaotic systems in the high-dimensional financial and economic systems and drew some useful conclusions. It has drawn much more attention to scholars because of its potential applications in synchronization, securer communications, and so on, and it 
been studied by Yu et al. $[18,19]$. Thus, there are important theoretical and practical meanings and wide foreground to further pursue this research.

Chaos and hyperchaos make a tremendous negative impact on the practice, so we can join disturbance or control in the system to delay the happening time of chaos. It is well known that there are many methods to control the chaotic and hyperchaotic systems, such as nonlinear feedback, speed feedback control, state feedback control [20], linear feedback control [21], and adaptive control. There are advantages and disadvantages in each control method. In this case, we should choose the correct method to achieve the goal of chaos and hyperchaos control based on the first control.

It is well known that when a state feedback control join into a three-dimension chaotic system, the original threedimension chaotic systems achieve a steady state by changing the value of the control parameters at the same time the original system goes into a four-dimensional system. However, through the theory analysis and numerical simulation, we found that the new four-dimension system produces both chaos and hyperchaos under the condition of certain ranges of parameters. This paper makes further analysis on chaotic and hyperchaotic system based on the new four-dimension system which has been joined in the state feedback control.

Thus, the new system which generates both chaos and hyperchaos is presented, and the complex dynamic characters of the system are analyzed. We adopt the method for the control according to the following rules: firstly, it is well known that the sooner convergence of the chaotic or hyperchaotic system, the better convergence of the effect of the control; secondly, the chaos control of a system should have some practical meanings; last but not the least, the controllers adopted by us should be as simple as possible, because it will be easy for us to operate in the application. Motivated by the discussion above, the linear control is proposed to achieve a second control, and it can drive the new hyperchaos to chaos, the periodic orbit, or a steady state; similarly, it can also restore a chaotic state to the equilibrium point. We outline two innovations, and one of which is that we will analyze and compare both chaotic and hyperchaotic Lyapunov dimensions and give the corresponding chaotic attractors and hyperchaotic attractors; the other one is that we introduce a second controller to the chaotic or hyperchaotic system which has already controlled by introducing a state feedback controller to delay or control hyperchaos, realizing the goal of a second control.

The rest of the paper is organized as follows. In Section 2, a four-dimension system is introduced, and we know that the system can produce both chaos and hyperchaos through numerical simulations. In Section 3, the system is analyzed dynamically, including the chaotic and hyperchaotic attractors. In Section 4, the power spectrums and time charts are mapped via Lyapunov dimension, Lyapunov exponents, and bifurcation diagram. In Section 5, linear feedback controller is designed for driving the chaotic or hyperchaotic system to a steady state, and the purpose of a second control is achieved. In the last section, the results are summarized, and the future directs are indicated.

\section{Construction of the Dynamic System}

In this paper, the dynamic system which has already introduced a state feedback control to the first equation is proposed by Correia and Rech [16], and the system of four-dimensional autonomous differential equations can be described as follows:

$$
\begin{gathered}
\dot{x}=a(x-y)-y z+w, \\
\dot{y}=-b y+x z, \\
\dot{z}=-c z+d x+x y, \\
\dot{w}=-e(x+y),
\end{gathered}
$$

where $a, b, c, d$, and $e$ are the parameters of system (1). When parameters $a=0.98, b=9, c=50, d=0.06$, and $e=0.9$ with the initial values $x=11.28, y=-11.21, z=-9$, and $w=$ 20.49 , the four Lyapunov exponents of system (1) calculated by Wolf algorithm are

$$
\begin{aligned}
L 1=0.007332, & L 2=0.004414, \\
L 3=-0.020359, & L 4=-0.898567 .
\end{aligned}
$$

As well as the detailed theoretical analysis, it has been confirmed that the new system (1) displays sophisticated and abundant hyperchaotic dynamical behaviors with parameters $a=0.98, b=9, c=50, d=0.06$, and $e=0.9$. The chaotic and hyperchaotic attractors which describe the dynamical behaviors of the system are shown in Figures 1, 2, and 3.

\section{Dynamics Analysis of the System}

In this section, basic properties and complex dynamics of the new system (1) are investigated, such as Lyapunov exponents, fractal dimensions, and attractors. The new dynamic system has the following basic properties.

3.1. Symmetry. The dynamic system (1) which is invariant under the coordinate transformation $(x, y, z, w) \rightarrow(-x,-y$, $z,-w)$ under reflection about the $z$-axis; the symmetry persists for all values of the system parameters.

3.2. Dissipation and Attractor. For the dynamical system (1), we can obtain

$$
\nabla V=\frac{\partial \dot{x}}{\partial x}+\frac{\partial \dot{y}}{\partial y}+\frac{\partial \dot{z}}{\partial z}+\frac{\partial \dot{w}}{\partial w}=a-b-c=m
$$

If we want the hyperchaotic system to become dissipative with $a-b-c=m$, then $m$ which is a negative value will be required. Dynamical system which is described by system (1) is one dissipative system. It is worth noting that a volume element $V_{0}$ is apparently contracted by the flow into a volume element $V_{0} e^{(a-b-c) t}$ in time $t$. From a mathematic point of view, it means that each volume containing the trajectory of this dynamical system shrinks to zero as $t$ at an exponential rate $a-b-c=m$. Therefore, all the orbits are ultimately confined to a specific subset of zero volume, and trajectories of the system arrive to an attractor at last. 
3.3. Equilibrium and Stability. The following equations are the equilibrium of the system satisfied:

$$
\begin{gathered}
a(x-y)-y z+w=0, \\
-b y+x z=0, \\
-c z+d x+x y=0, \\
-e(x+y)=0 .
\end{gathered}
$$

By calculation, there are three equilibrium points for the system, and the equilibrium points are as follows:

$E 1(0,0,0,0), E 2\left(-\left(d / 2+\sqrt{d^{2}+4 b c} / 2\right)(2 a-b), d / 2+\right.$ $\left.\sqrt{d^{2}+4 b c} / 2,-d / 2-\sqrt{d^{2}+4 b c} / 2,-b\right), \quad E 3(-(d / 2 \quad-$ $\left.\sqrt{d^{2}+4 b c} / 2\right)(2 a-b), d / 2-\sqrt{d^{2}+4 b c} / 2, \sqrt{d^{2}+4 b c} / 2-$ $d / 2,-b)$.

The Jacobian matrix evaluated at the three equilibrium points of the system can be expressed as follows:

$$
J=\left(\begin{array}{cccc}
a & -a-z & -y & 1 \\
z & -b & x & 0 \\
d+y & x & -c & 0 \\
-e & -e & 0 & 0
\end{array}\right)
$$

It is easy to get the characteristic equation $|\lambda E-J|=0$. Thus, we can obtain the eigenvalues at the equilibrium points by solving the characteristic equation. When

$$
\begin{gathered}
a=0.98, \quad b=9, \quad c=50, \\
d=0.06, \quad e=0.9,
\end{gathered}
$$

the characteristic equation for equilibrium point $E 2$ has four eigenvalues, and it is easy to show that the four eigenvalues are

$$
\begin{gathered}
\lambda_{1}=-51.5250, \quad \lambda_{2}=-3.2647+10.8594 i, \\
\lambda_{3}=-3.2647-10.8594 i, \quad \lambda_{4}=0.0344 .
\end{gathered}
$$

Obviously, $\lambda_{1}$ is a negative number, $\lambda_{2}$ and $\lambda_{3}$ are a pair of complex conjugate eigenvalues with negative real parts, and the forth number $\lambda_{4}$ is a positive real number. Hence, the equilibrium point $E 2$ is an unstable saddle point in the system, and the hyperchaotic system is unstable at the point E2. At the same time, it is easy to prove that both the equilib-rium points $E 1$ and $E 3$ are also unstable saddle points.

3.4. Lyapunov Exponents and Lyapunov Dimensions. It is known that most of the dynamic systems can be charactered by the Lyapunov exponents. Figure 4 describes Lyapunov exponents with the variety of parameters $a$ and $b$ on the premise of other parameters fixed when the step length is 0.001 . Figure 4(a) is the Lyapunov exponents with the variety of $a$ when $a \in[1,2.6], b=12, c=5, d=0.06$, and $e=0.1$, and Figure 4(b) is the Lyapunov exponents with the varies of $b$ when $a=1, b \in[12,22], c=5, d=0.06$, and $e=0.1$. As it can be seen in Figure 4(a), there is one positive value in certain ranges of parameter $a$, so it is chaotic at this time. In some ranges of parameter $a$, there are two positive Lyapunov exponents and two negative exponents; the system rushes into a hyperchaotic state. In some ranges of parameter $a$, there is one zero and three negative Lyapunov exponents, and the dynamic system appears periodic. In some special points, there are two zero and two negative Lyapunov exponents, and the dynamic system appears quasiperiodic. From Figure 4(b), it is easy to see that it is similar to Figure 4(a). In one word, the diversity for the combination of Lyapunov exponents results in the dynamic system appearing more complex behaviors. According to the theory of chaos, the Lyapunov exponents measure the exponential rates of divergence and convergence of nearby trajectories in phase space of dynamic system (1) [22-24]. As we know that for a chaotic attractor, one positive, one zero, and two negative Lyapunov exponents are needed in a dynamic system, while for the corresponding hyperchaotic attractors, the system should have two positive Lyapunov exponents at least.

3.4.1. Chaos. For the system when $a=1, b=12, c=5$, $d=0.06$, and $e=0.1$, the four Lyapunov exponents are as follows:

$$
\begin{aligned}
L 1=0.003202, & L 2=-0.000341, \\
L 3=-0.003468, & L 4=-0.173814 .
\end{aligned}
$$

It can be seen that the largest Lyapunov exponent is positive, indicating that the system has chaotic characteristics. The $L 1$ is a positive Lyapunov exponent, and the rest three Lyapunov exponents are negative. Thus, the system is chaotic, not hyperchaotic. The fractal dimension is also a typical characteristic of chaos calculated Kaplan-Yorke dimension by Lyapunov exponents, and $D_{k y}$ can be expressed as

$$
D_{k y}=k+\sum_{i=1}^{k} \frac{L_{i}}{\left|L_{k+1}\right|} \text {, }
$$

where $k$ says the first $k$ Lyapunov exponent is nonnegative, namely, $k$ is the maximum value of $i$ value which meets both $\sum_{i=1}^{k} L_{i} \gg 0$ and $\sum_{i=1}^{k} L_{i}<0$ at the same time. $L_{i}$ is in descending order of the sequence according to the sequence of Lyapunov exponents. $D_{k y}$ is the upper bound of the dimension of the system information. For the system in this paper, by observing the values of four Lyapunov exponents in the above, we determine that the value of $k$ is two, and then the Kaplan-Yorke dimension can be expressed from the above

$$
D_{k y}=2+\frac{0.003202-0.000341}{|-0.003468|}=2.824971 \approx 2.825 \text {. }
$$

So, chaos in this system is very obvious. Thus, the corresponding attractors are shown in Figure 5.

3.4.2. Hyperchaos. When $a=1, b=12, c=50, d=0.06$, and $e=0.9$ and initial values are $x=11.28, y=-11.21, z=-9$, and $w=20.49$, the Lyapunov exponents are as follows:

$$
\begin{array}{cl}
L 1=0.007048, & L 2=0.004036, \\
L 3=-0.070032, & L 4=-0.855856 .
\end{array}
$$




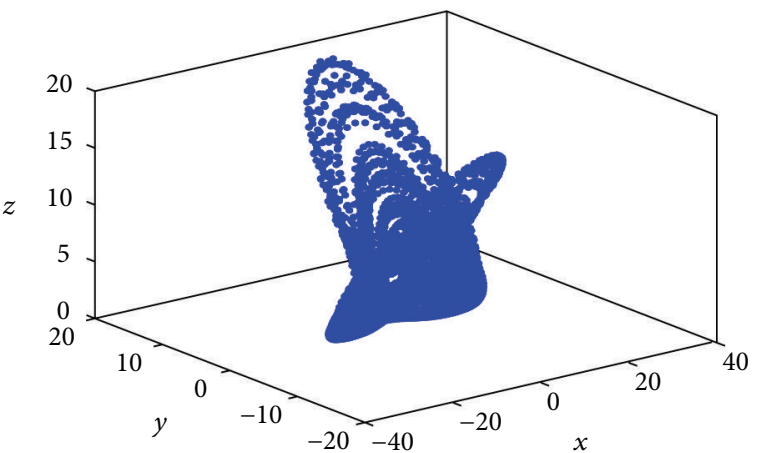

(a) $3 \mathrm{D}$ view in the $x-y-z$ space

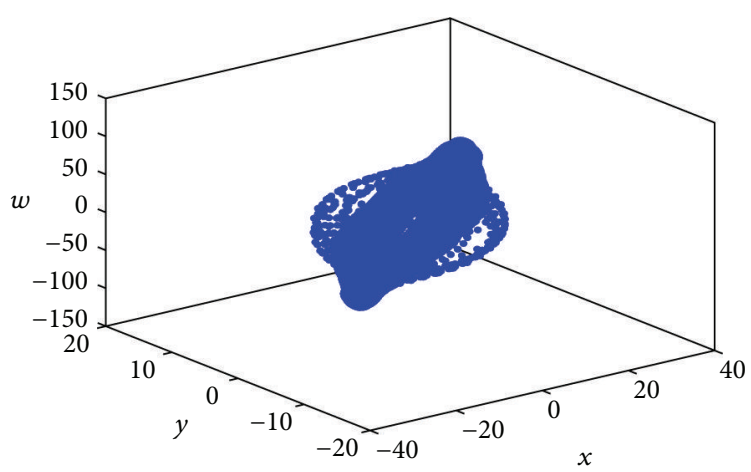

(c) $3 \mathrm{D}$ view in the $x-y-w$ space

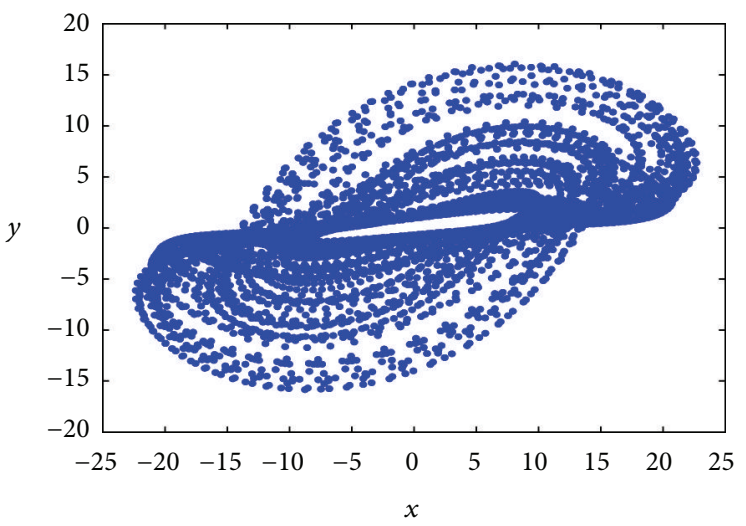

(e) Projection onto the $x-y$ plane

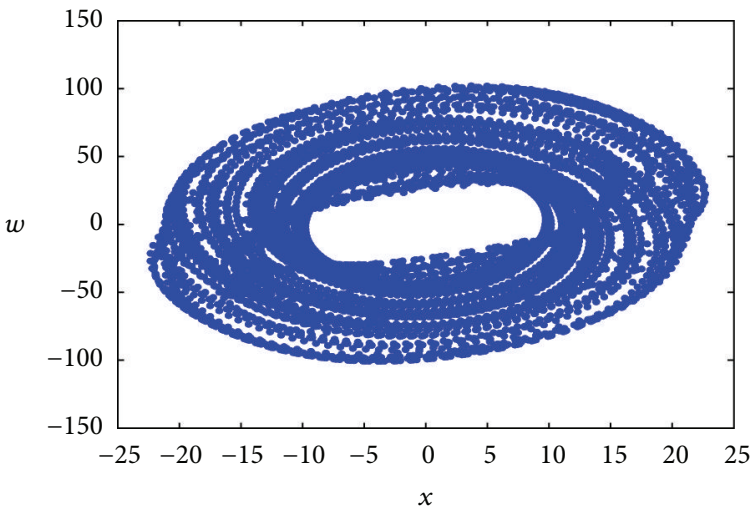

(g) Projection onto the $x-w$ plane

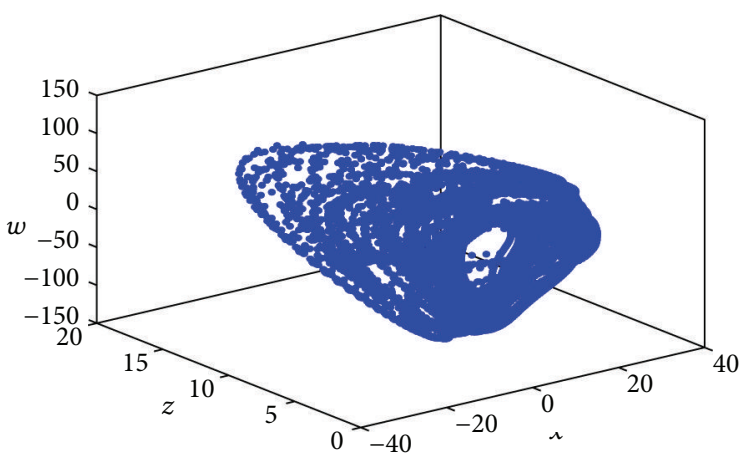

(b) $3 \mathrm{D}$ view in the $x-z-w$ space

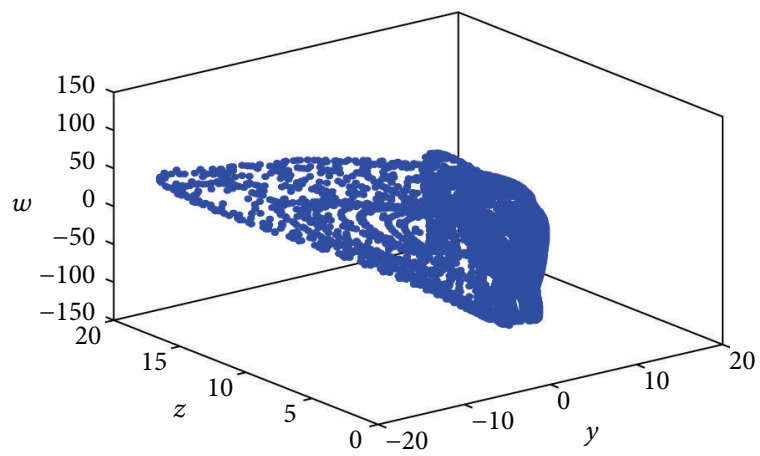

(d) $3 \mathrm{D}$ view in the $y-z-w$ space

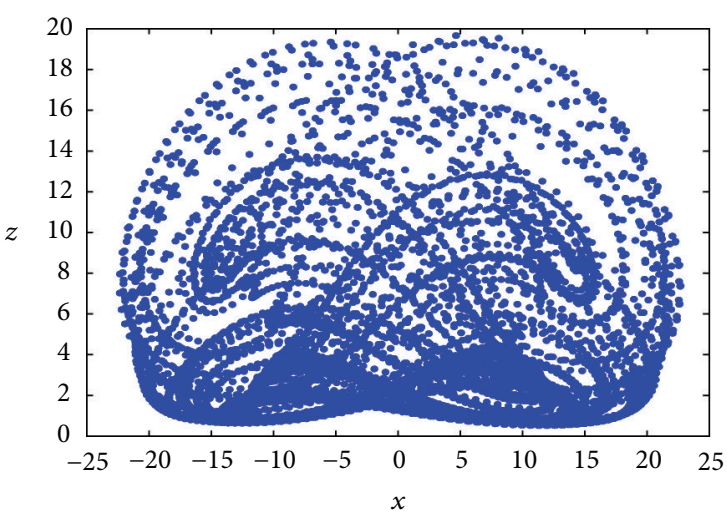

(f) Projection onto the $x$ - $z$ plane

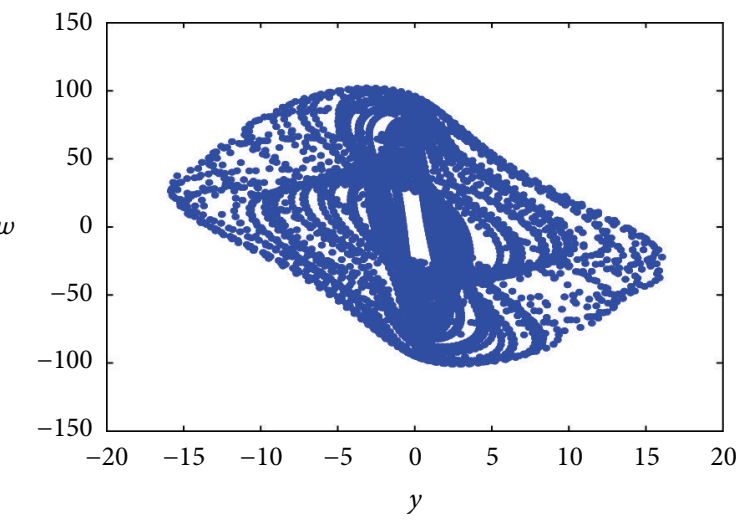

(h) Projection onto the $y$ - $w$ plane

Figure 1: Continued. 


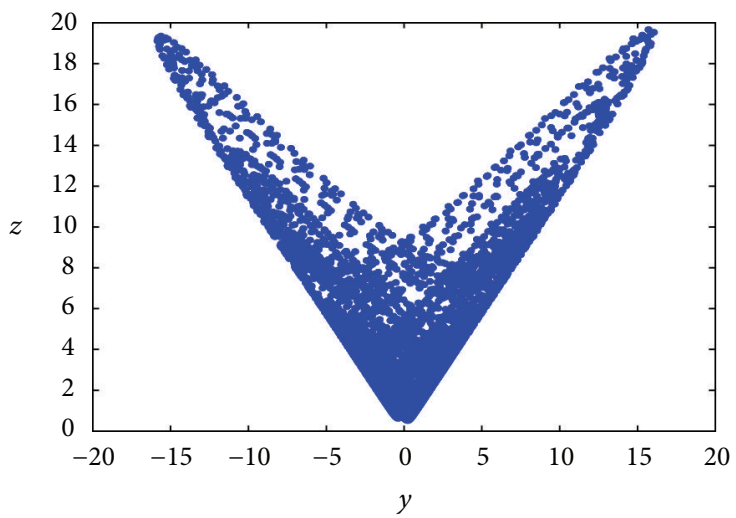

(i) Projection onto the $y$ - $z$ plane

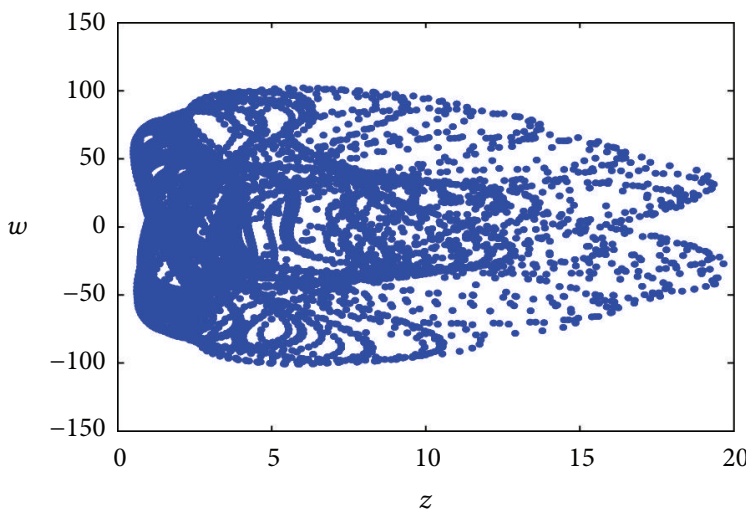

(j) Projection onto the $z-w$ plane

Figure 1: Phase portraits of the dynamic system when $x=11.28, y=-11.21, z=-9$, and $w=20.49$ and $a=0.9, b=12, c=6, d=0.06$, and $e=20$.

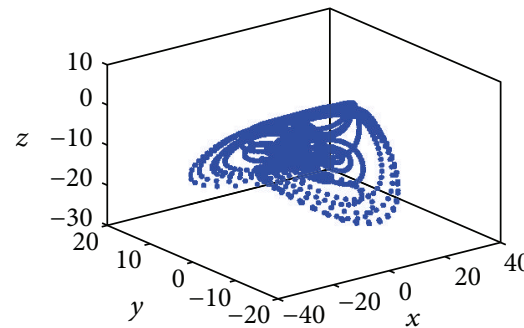

(a) $3 \mathrm{D}$ view in the $x-y-z$ space

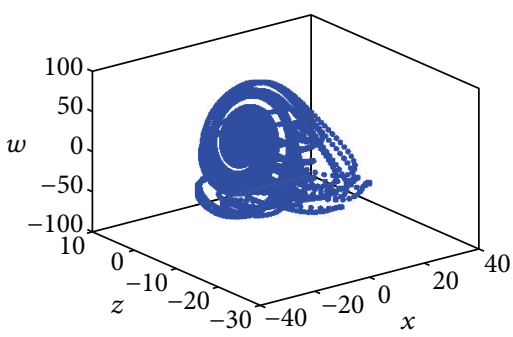

(d) $3 \mathrm{D}$ view in the $x-z-w$ space

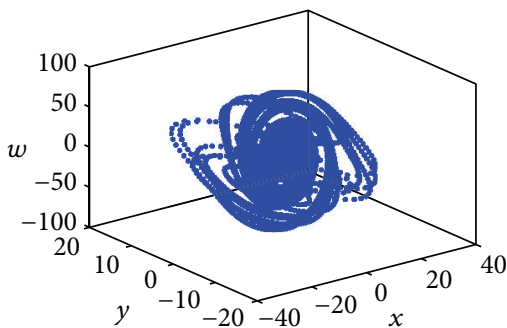

(b) $3 \mathrm{D}$ view in the $x-y-w$ space

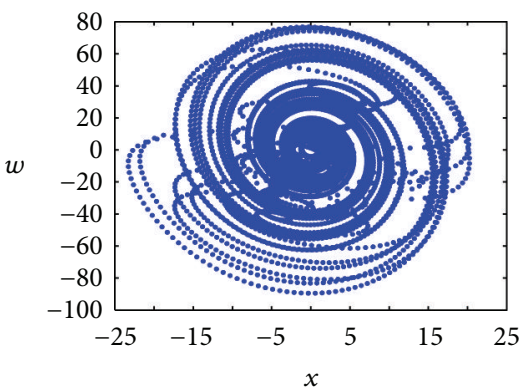

(e) Projection onto the $x-w$ plane

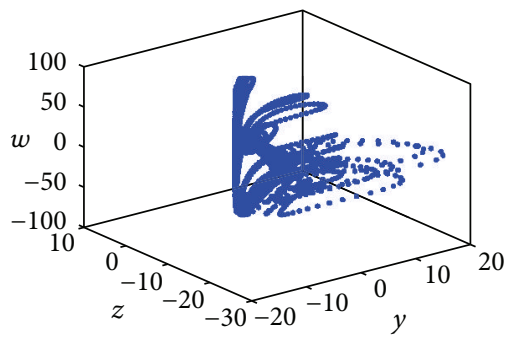

(c) $3 \mathrm{D}$ view in the $y-z-w$ space

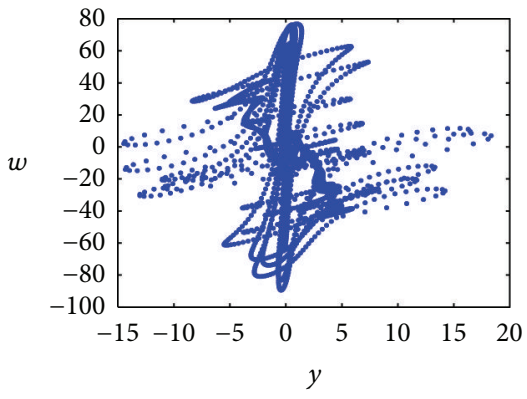

(f) Projection onto the $y$ - $w$ plane

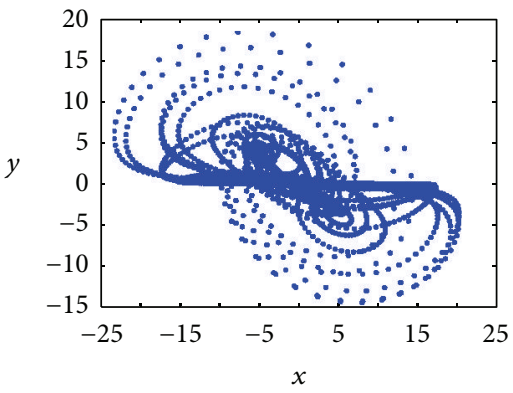

(g) Projection onto the $x-y$ plane

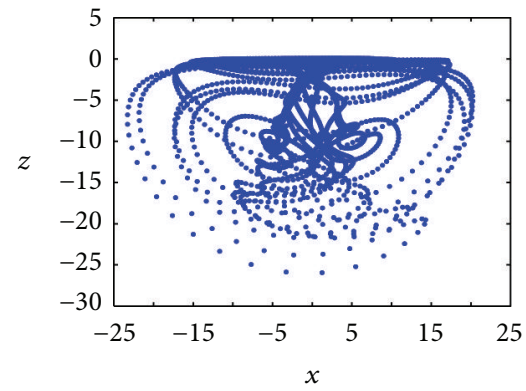

(h) Projection onto the $x-z$ plane

FIgUre 2: Continued. 


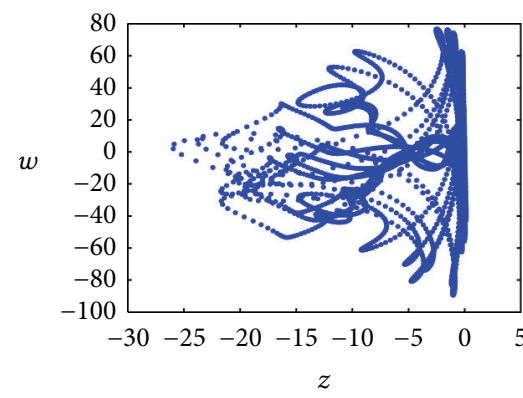

(i) Projection onto the $z-w$ plane

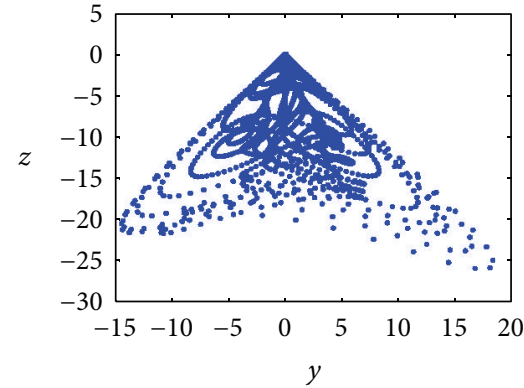

(j) Projection onto the $y$ - $z$ plane

Figure 2: Phase portraits of the dynamic system when $x=11.28, y=-11.21, z=-9$, and $w=20.49$ and $a=0.9, b=12, c=2, d=0.06$, and $e=20$.

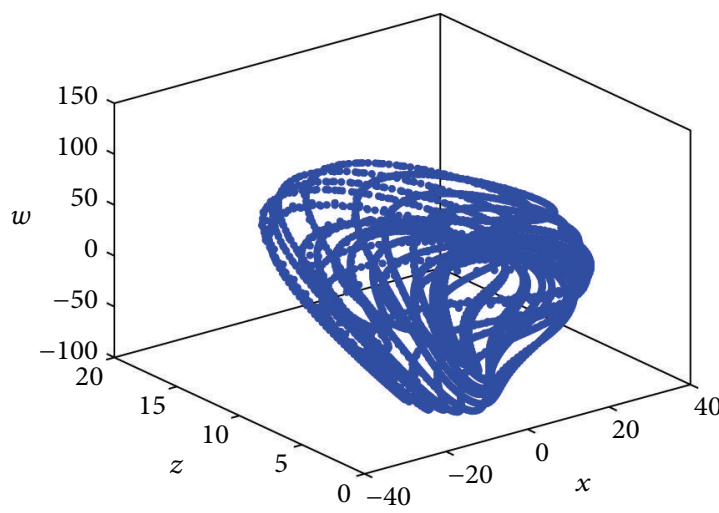

(a) Attractors when $a=0.9, b=12, c=6.7, d=$ 0.06 , and $e=20$

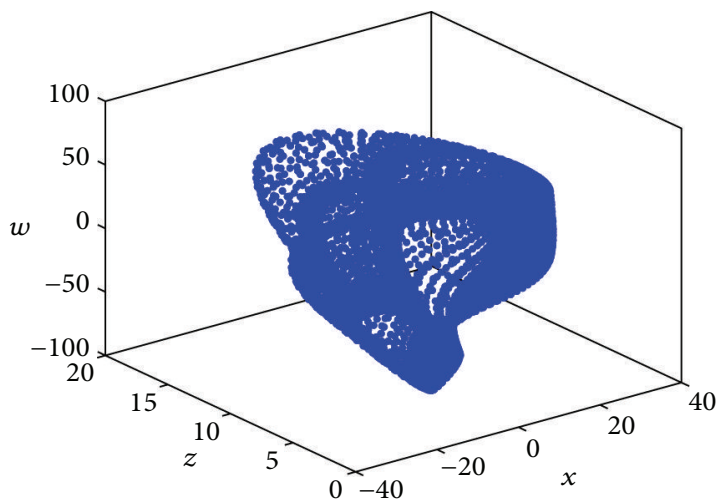

(c) Attractors when $a=0.9, b=12, c=7.9, d=$ 0.06 , and $e=20$

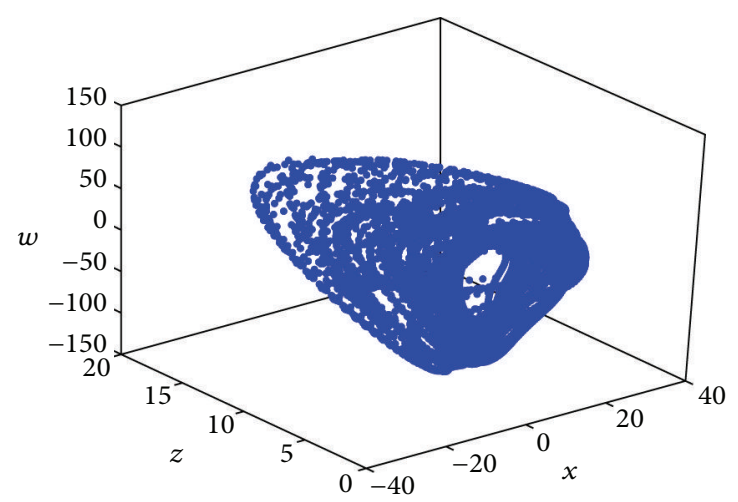

(b) Attractors when $a=0.9, b=12, c=6.7, d=$ 0.06 , and $e=20$

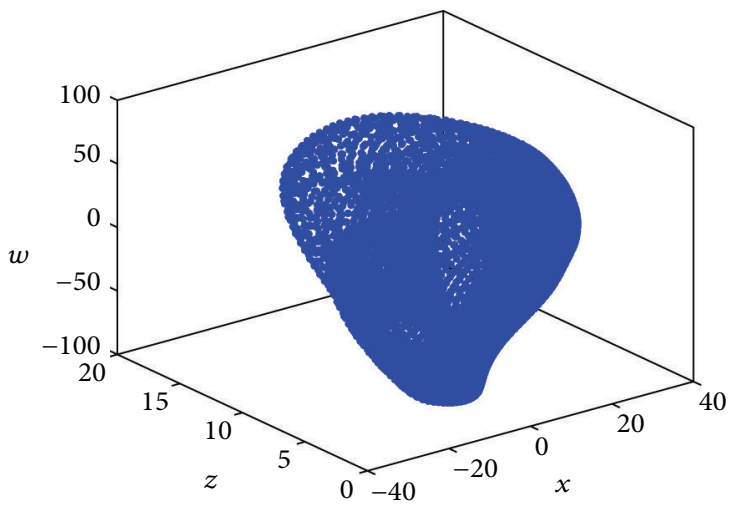

(d) Attractors when $a=0.98, b=12, c=6, d=0.06$, and $e=$ 24

FIGURE 3: Attractors when $x=11.28, y=-11.21, z=-9$, and $w=20.49$ with different parameters.

The fractal dimension can be written as follows:

$$
D_{k y}=2+\frac{0.007048-0.004036}{|-0.070032|}=2.0430089 \approx 2.04 \text {. }
$$

As is well known, there is more than one positive exponent in the four-dimensional dynamic system. For the system which has been controlled by a state feedback controller, it is chaotic within certain ranges of parameters. However, the system will rush into a hyperchaotic state when there are two or more than two positive Lyapunov exponents. Thus, hyperchaotic attractors also exist in the system as it can be seen from Figure 6, and the behaviors of system will become much more complex. Therefore, both chaos and hyperchaos appear very obviously.

\section{Bifurcation Diagrams}

Generally speaking, the bifurcation diagram is the best method to describe all the possible behaviors as the parameter varies in one diagram. We take 500 as the total number of time 


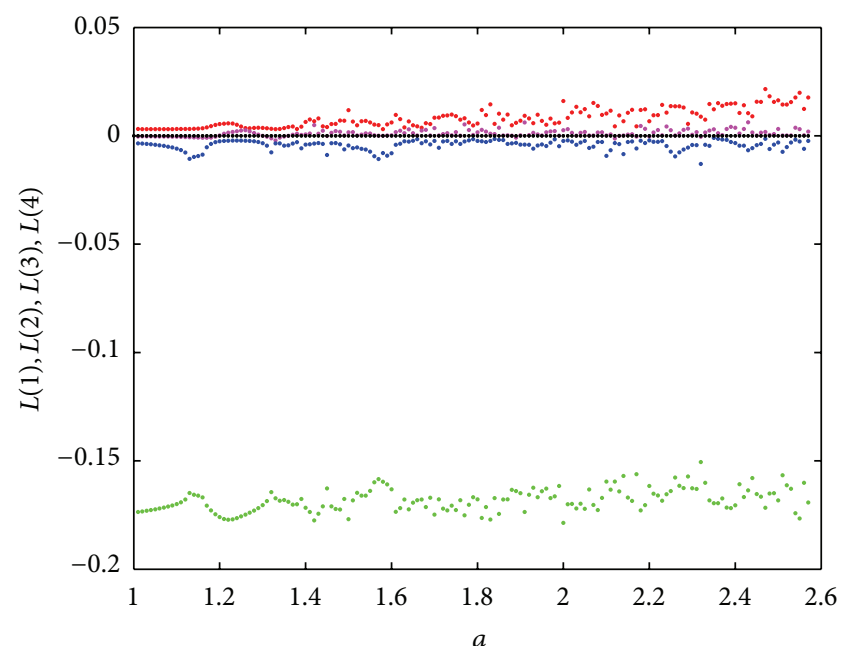

(a) The Lyapunov exponents with the variety of $a$, when $b=12, c=5, d=$ 0.06 , and $e=0.1$

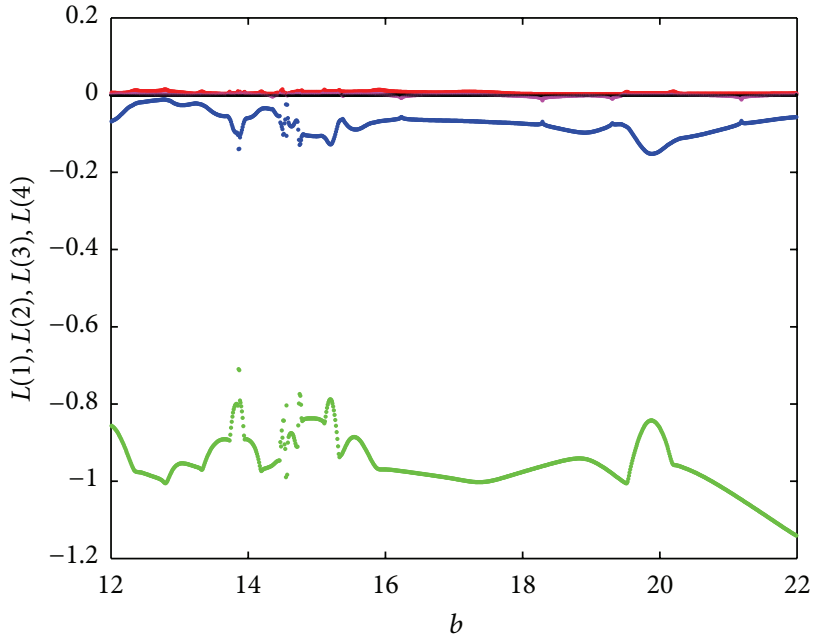

(b) The Lyapunov exponents with the variety of $b$, when $a=1, c=$ $5, d=0.06$, and $e=0.1$

FIgURE 4: The Lyapunov exponents with the variety of parameters $a(a \in[1,2.6])$ and $b(b \in[12,22])$ when the step length is 0.001 .

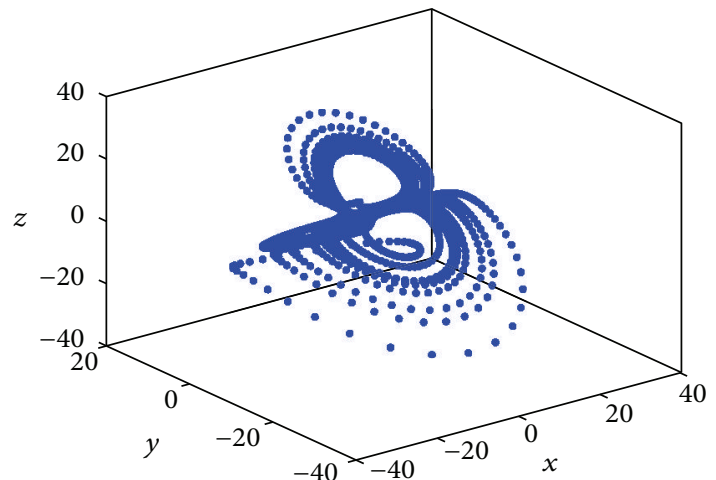

(a) $3 \mathrm{D}$ view in the space $x-y-z$

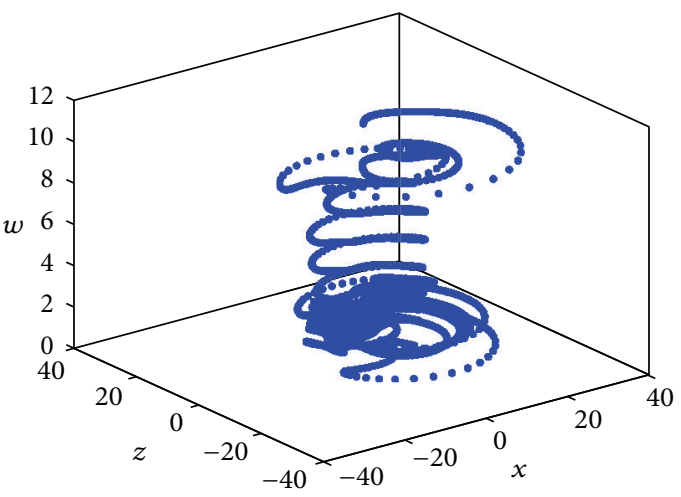

(c) $3 \mathrm{D}$ view in the space $x-z-w$

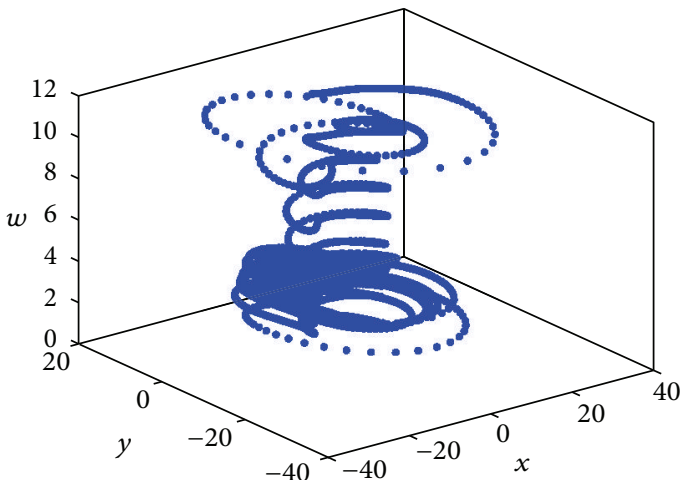

(b) $3 \mathrm{D}$ view in the space $x-y-w$

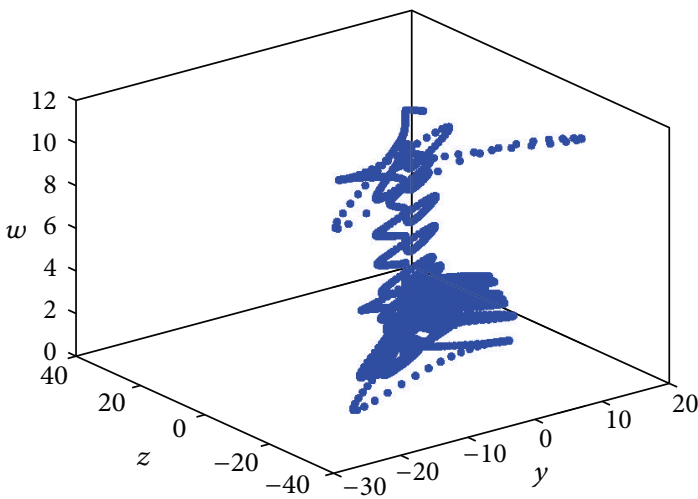

(d) $3 \mathrm{D}$ view in the space $y-z-w$

FIGURE 5: Chaotic attractors.

steps, 400 as the number of time steps discarded, and 0.001 as the step length, calculating and drawing the bifurcation diagram according to the definition of dynamical system. We map out the bifurcation diagrams with the variety of parameters $a, b, c, d$, and $e$, respectively, $(a \in[-3,3], b \in$ $[0,8], c \in[-1,6], d \in[20,30]$, and $e \in[33,43])$ in
Figures $7(\mathrm{a})-7(\mathrm{e})$. The bifurcation diagram of system (1) shows the complicated bifurcation phenomena. In order to make a detailed description of the behaviors of system, we map out the Lyapunov exponents with the variety of $a,(a \in$ $[-3,0.6])$ as an example in Figure $7(f)$. Thus, we can learn about the dynamic behaviors furthermore by comparing the 


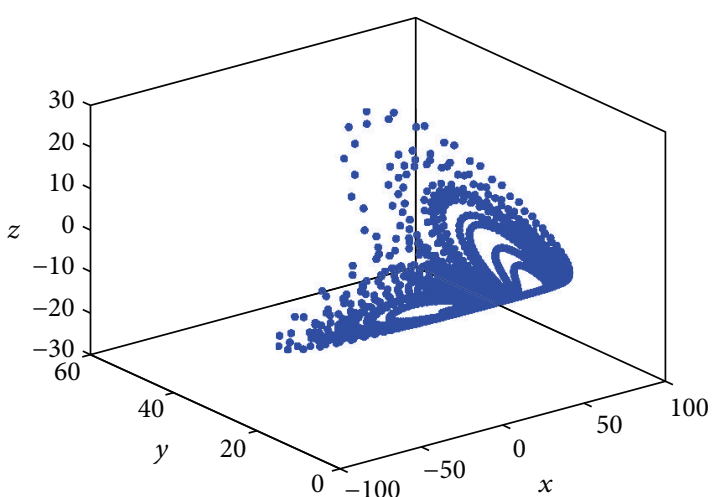

(a) $3 \mathrm{D}$ view in the space $x-y-z$

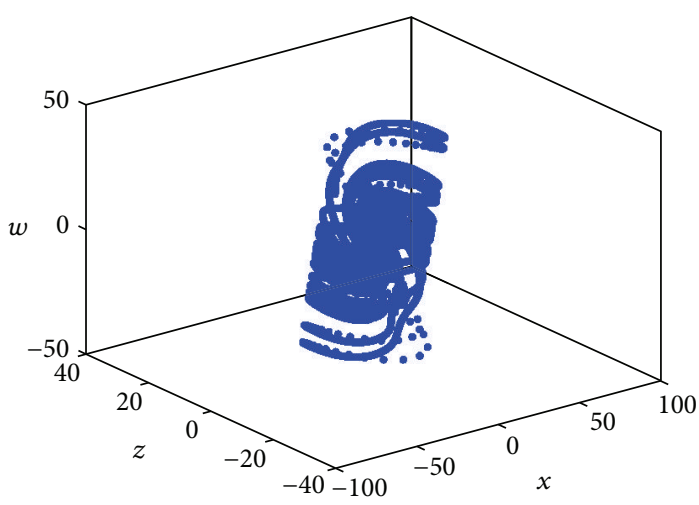

(c) $3 \mathrm{D}$ view in the space $x-z-w$

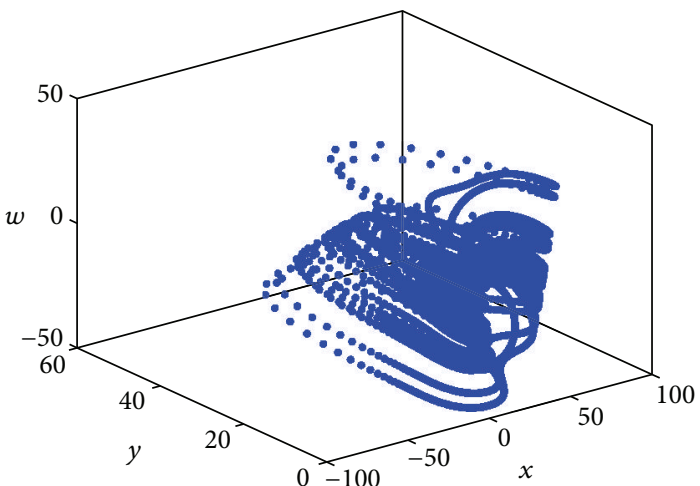

(b) $3 \mathrm{D}$ view in the space $x-y-w$

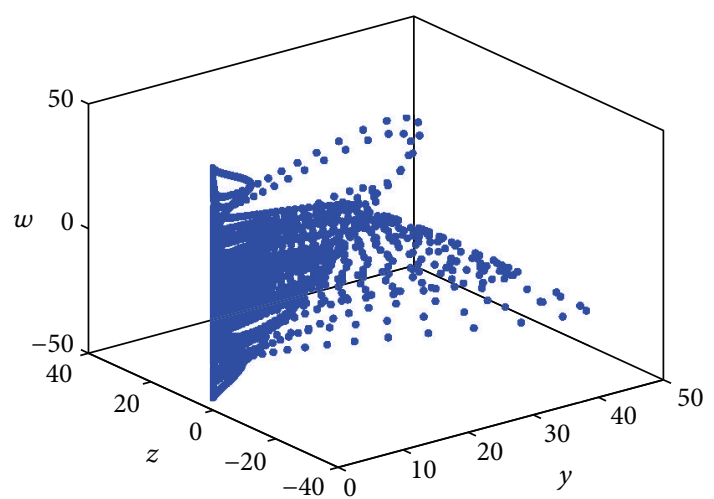

(d) $3 \mathrm{D}$ view in the space $y-z-w$

FIGURE 6: Hyperchaotic attractors.

bifurcation diagram and the Lyapunov exponents in different stages. Attractors are also mapped out in every stage as it can be seen in Figure 8 .

Comparing Figure 7(a) with 7(f), it is easy to see that the two figures are consistent. When $b=9, c=5, d=0.06$, and $e=25$ and step length is 0.001 , the values of $a=-1.59$ and $a=-0.56$ divide the system into several stages. We assume that four Lyapunov exponents of system (1) satisfying $L_{1}>$ $L_{2}>L_{3}>L_{4}$ and the dynamical behaviors can be described and classified as follows

(1) For $a<-1.59, L_{1}<0, L_{2}<0, L_{3}<0$, and $L_{4}<0$, the system (1) is in a steady state and stabilized to the equilibrium point.

(2) For $a=-1.59, L_{1}=0, L_{2}<0, L_{3}<0$, and $L_{4}<0$, the system (1) appears periodic, because there is one zero and three negative Lyapunov exponents; Figures $8(\mathrm{a})-8(\mathrm{~d})$ show the corresponding attractors in this stage.

(3) For $-1.59<a<-0.56, L_{1}>0, L_{2}<0, L_{3}<0$, and $L_{4}<0$, one positive Lyapunov exponent exists, and the system (1) is in a chaotic state at this time; Figures $8(\mathrm{e})-8(\mathrm{~h})$ show the corresponding chaotic attractors in this stage.

(4) For $a=-0.56, L_{1}>0, L_{2}=0, L_{3}<0$, and $L_{4}<0$, the system (1) is still in a chaotic state, because there is one positive Lyapunov exponent, one zero Lyapunov exponent, and two negative Lyapunov exponents; Figures 8(i)-8(l) show the corresponding chaotic attractors in this stage.

(5) For $a>-0.56, L_{1}>0, L_{2}>0, L_{3}<0$, and $L_{4}<0$, two positive Lyapunov exponents exist, and the system (1) rushes into a hyperchaotic state, and hyperchaotic attractor appears. Figures $8(\mathrm{~m})$ $8(\mathrm{p})$ show hyperchaotic attractors in this stage.

4.1. The Power Spectrum of the System. When $x=11.28$, $y=-11.21, z=-9$, and $w=20.49$, Figure 9 is the power spectrums of the system with different parameters. Figure 9(a) is the power spectrums of the dynamic system with $a=0.98, b=12, c=6, d=0.06$, and $e=20$. Figure 9(b) is the power spectrums of the dynamic system with $a=0.9, b=12, c=6, d=0.06$, and $e=20$. Figure 9(c) is the power spectrums of the dynamic system with $a=0.9, b=12, c=6, d=0.06$, and $e=25$. Figure 9 (d) is the power spectrums of the dynamic system when $x=$ 11.28, $y=-11.21, z=-9$, and $w=20.49$. According to numerical simulation of the system, take the cycle method to estimate the power spectrums of variables of the system, as it is shown in Figures 9(a)-9(d) with different parameters. According to the numerical calculation, the results show that with parameters changing, the values of the system have always been restricted in a certain range. This proves the 


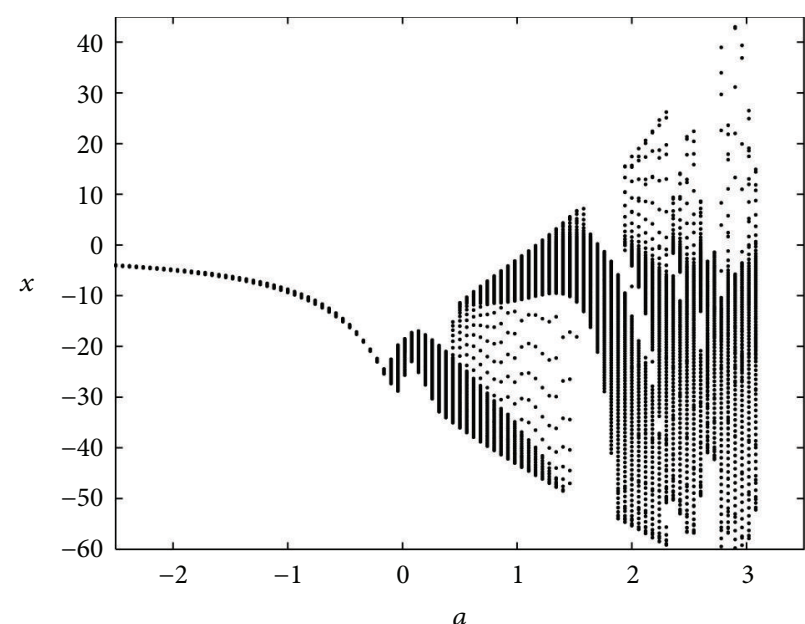

(a) Bifurcation diagram with the change of $a$, when $b=9, c=5, d=$ 0.06 , and $e=25$

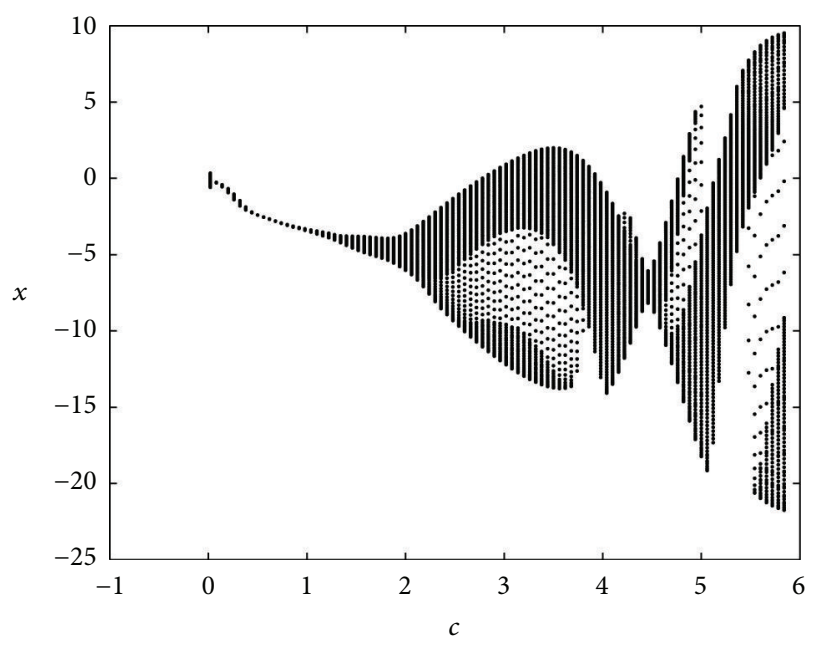

(c) Bifurcation diagram with the change of $c$ with the rest fixed parameters

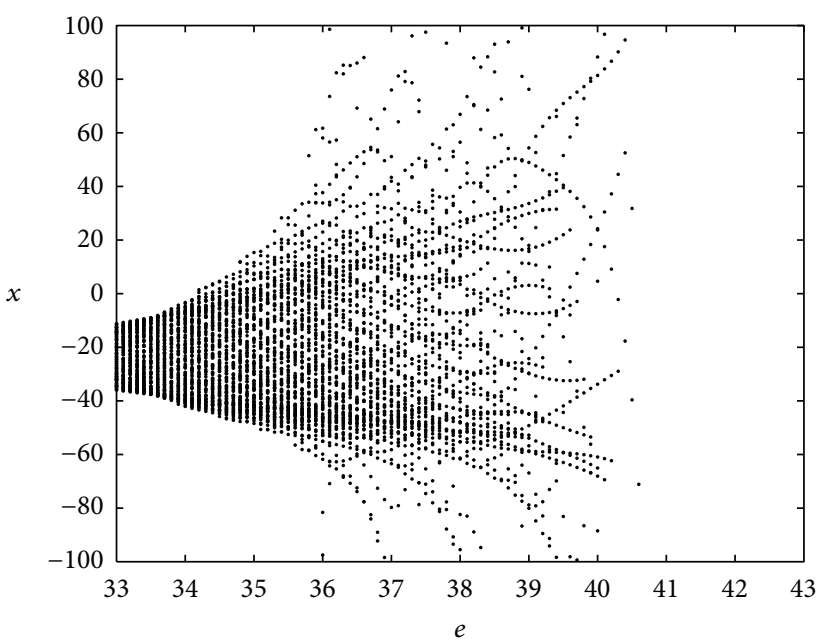

(e) Bifurcation diagram with the change of $e$ with the rest fixed parameters

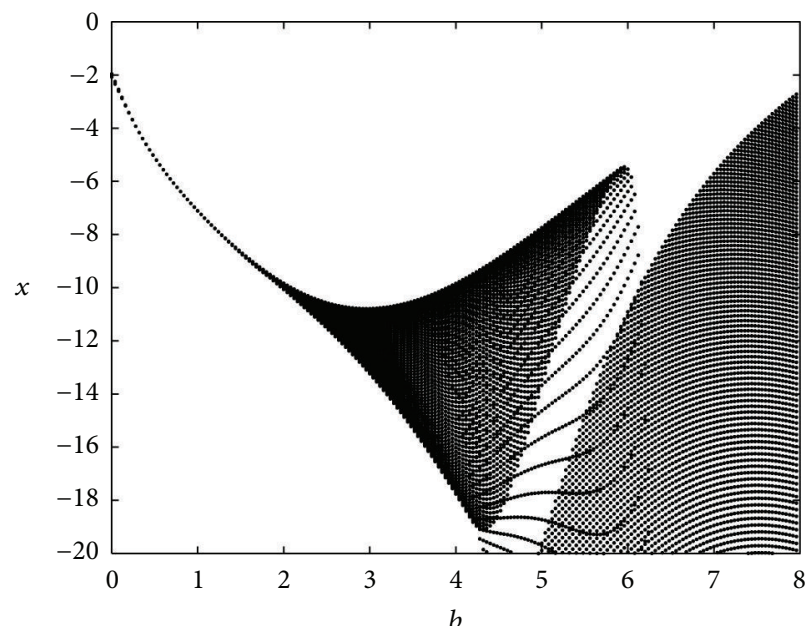

(b) Bifurcation diagram with the change of $b$ with the rest fixed parameters

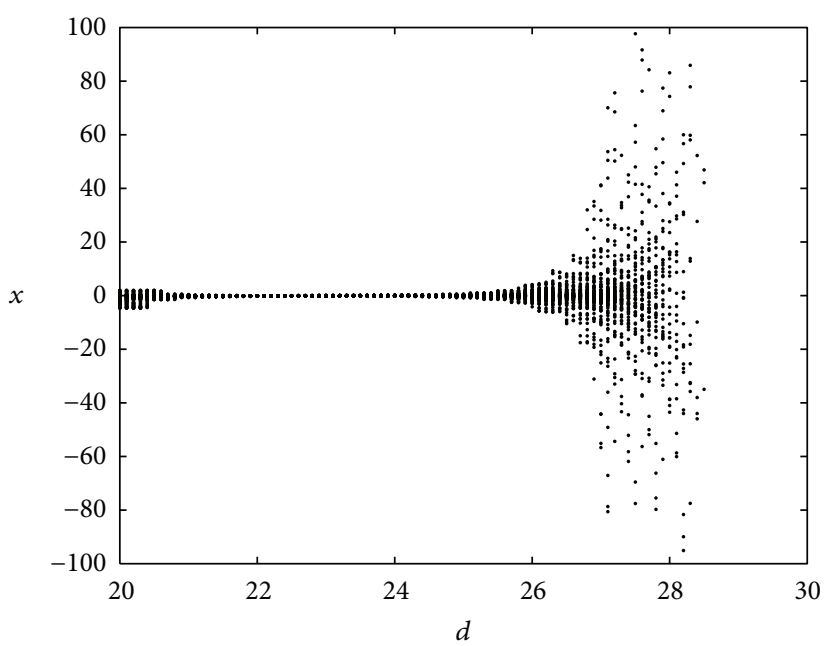

(d) Bifurcation diagram with the change of $d$ with the rest fixed parameters

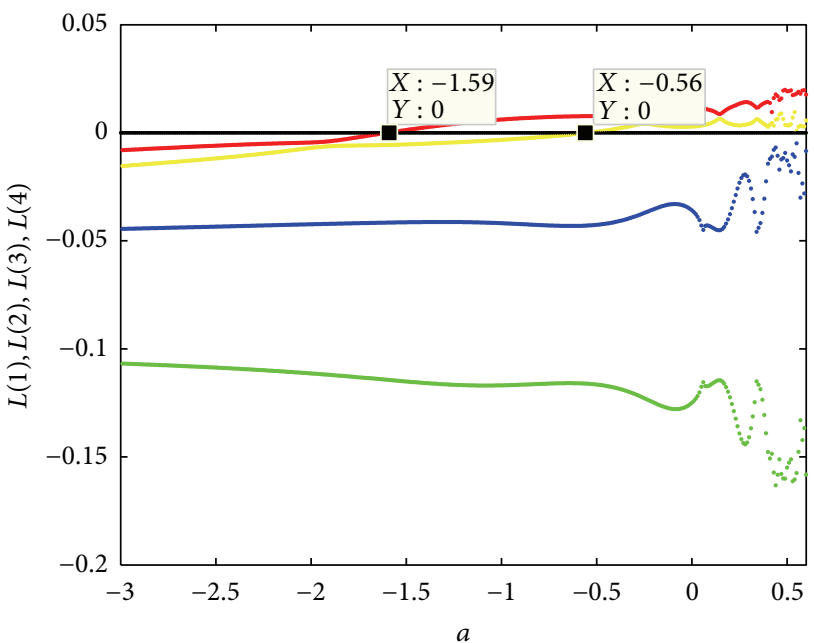

(f) Lyapunov exponent with the change of $a$, when $b=9, c=5, d=$ 0.06 , and $e=25$

FIGURE 7: Bifurcation diagram and the Lyapunov exponent when $x=11.28, y=-11.21, z=-9$, and $w=20.49$ and the step length is 0.001 . 


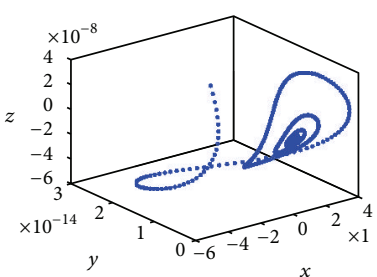

(a)

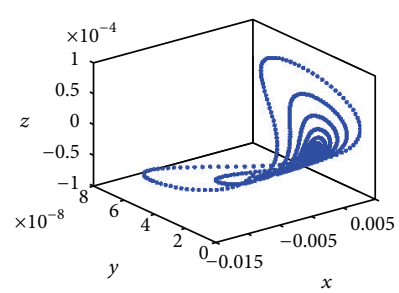

(e)

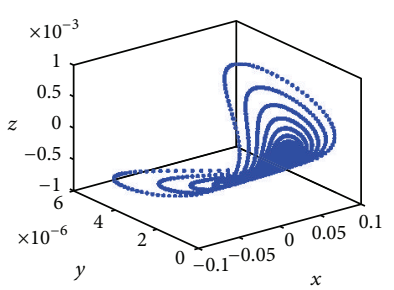

(i)

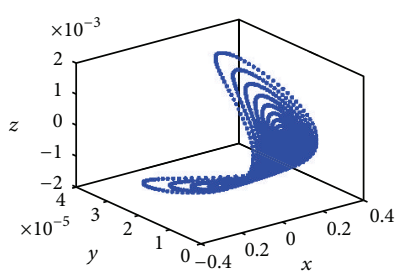

(m)

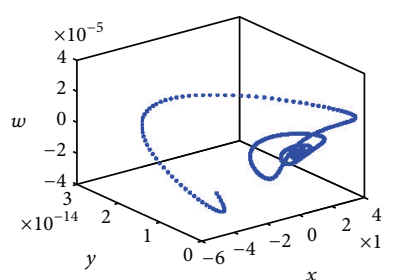

(b)

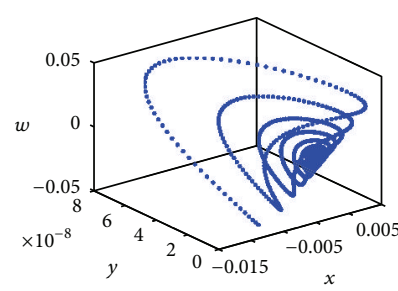

(f)

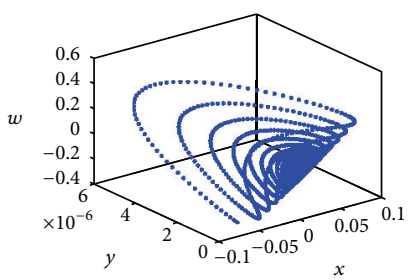

(j)

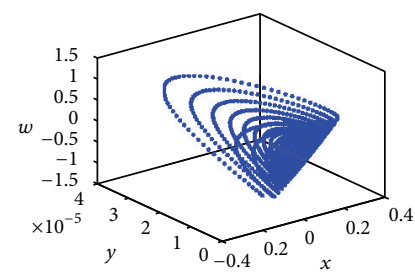

(n)

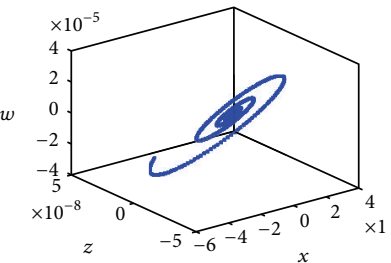

(c)

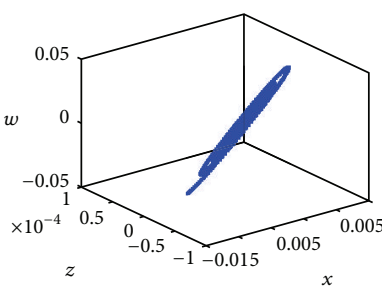

(g)

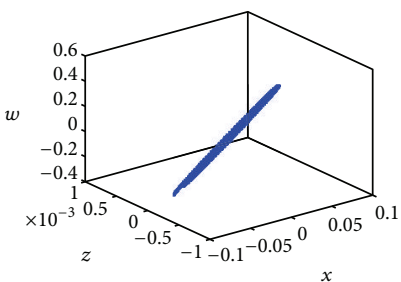

(k)

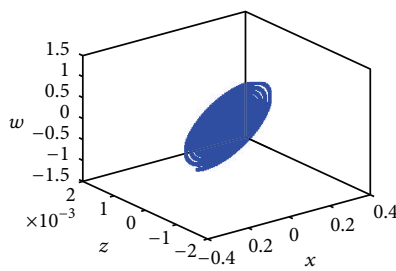

(o)

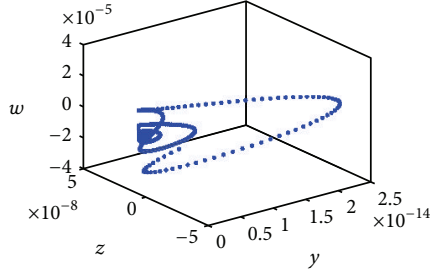

(d)

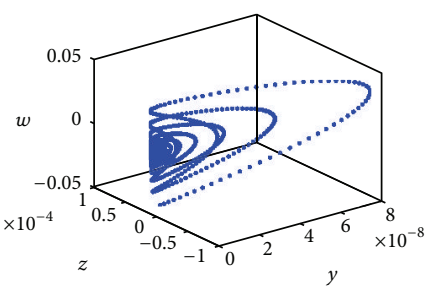

(h)

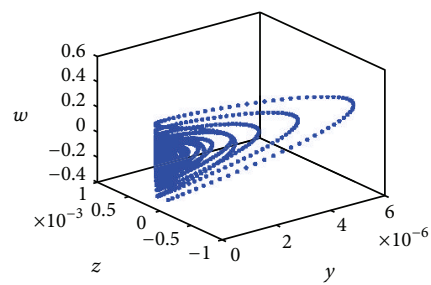

(l)

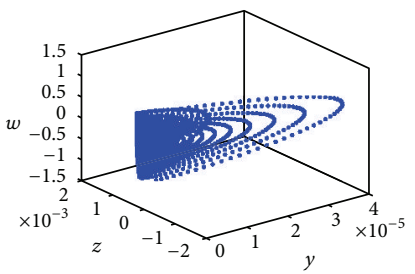

(p)

Figure 8: Typical dynamical behavior of system (1). (a)-(d): $a=-1.59,(\mathrm{e})-(\mathrm{h}): a=-1.5,(\mathrm{i})-(\mathrm{l}): a=-0.56$, and (m) $-(\mathrm{p}): a=0.2$.

characteristics of the chaotic system once again: boundedness and ergodicity. Therefore, there are some methods which can be taken to control the chaos and hyperchaos.

4.2. The Time Chart. The time chart is also a good method to display the behaviors as the parameter varies on the figure. It can be seen that the time charts of the system are shown in Figure 10 when $x=11.28, y=-11.21, z=-9$, and $w=20.49$ and $a=0.98, b=12, c=6, d=0.06$, and $e=20$.

\section{Chaos Control}

Chaos and hyperchaos may cause irregular behaviors which are undesirable, so not all the partners in the system can make correct strategies or reasonable decisions in the market. However, in some special environment, chaos and hyperchaos are helpful to our economy. Thus, proper measures should be taken to control chaos in order to advance or delay the occurrence of the chaos or hyperchaos. In this chapter, we take the linear feedback control method. The method comes from the differences between the target signal and output chaotic signal or directly uses the chaotic output signal of the system itself, multiplied by the appropriate coefficient of linear feedback. Several characteristics of this method are described as follows: (1) the method can achieve control for any solution in the original system, such as the fixed point and unstable periodic orbits; (2) it is not affected by the changes of the small parameter, with the characteristic of antiinterference; and (3) there are certain difficulties for the actual system which exist in the interaction of many system variables. Therefore, we should make further improvement from the point of view of engineering application. Nonfeedback and the delay feedback are two methods for the chaos control. Thus, we can achieve the task of chaos control in this dynamic system. Firstly, we have a transformation of the system at the equilibrium point $E 2$ as the following rules:

$$
\begin{gathered}
X=x+\left(\frac{d}{2}+\frac{\sqrt{d^{2}+4 b c}}{2}\right)(2 a-b), \\
Y=y-\left(\frac{d}{2}+\frac{\sqrt{d^{2}+4 b c}}{2}\right),
\end{gathered}
$$




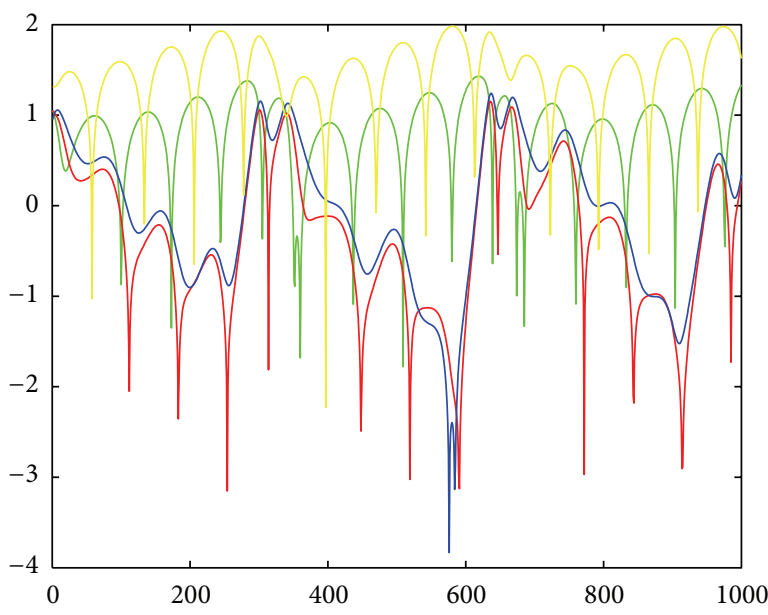

(a) The power spectrums of the dynamic system with $a=0.98, b=$ $12, c=6, d=0.06$, and $e=20$

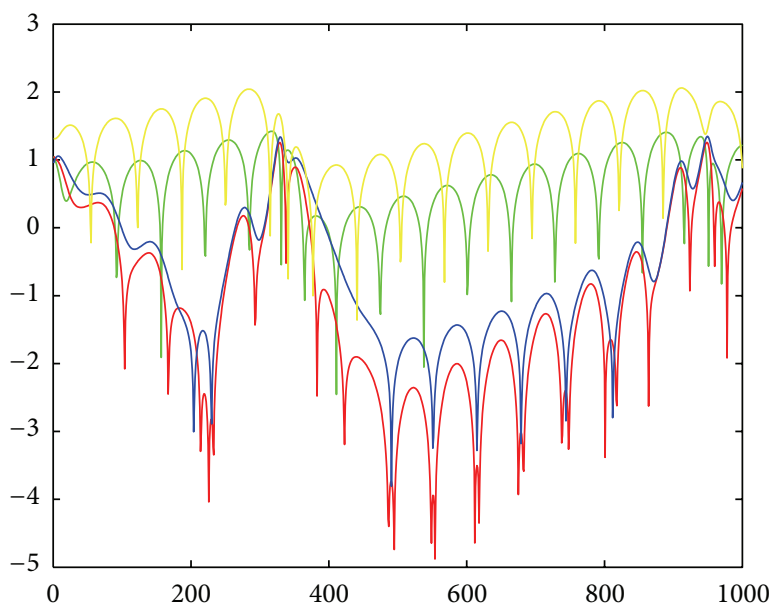

(c) The power spectrums of the dynamic system with $a=0.9, b=$ $12, c=6, d=0.06$, and $e=25$

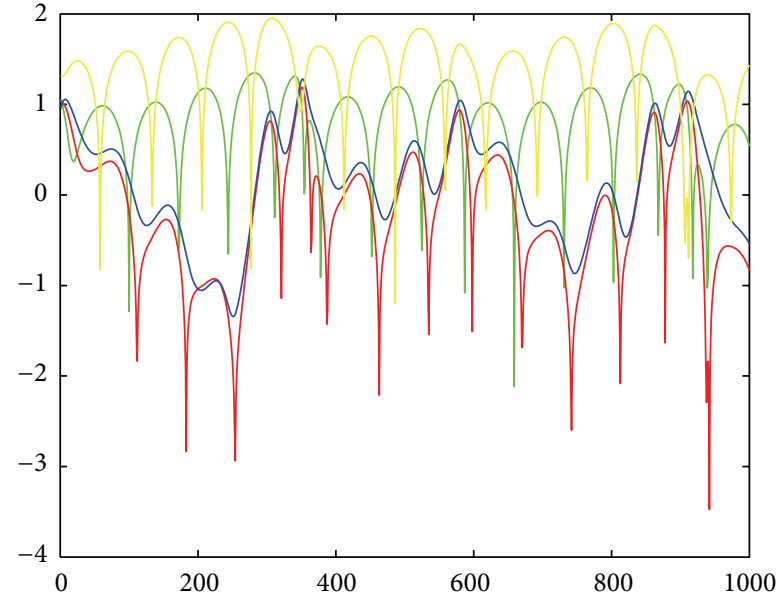

(b) The power spectrums of the dynamic system with $a=0.9, b=$ $12, c=6, d=0.06$, and $e=20$

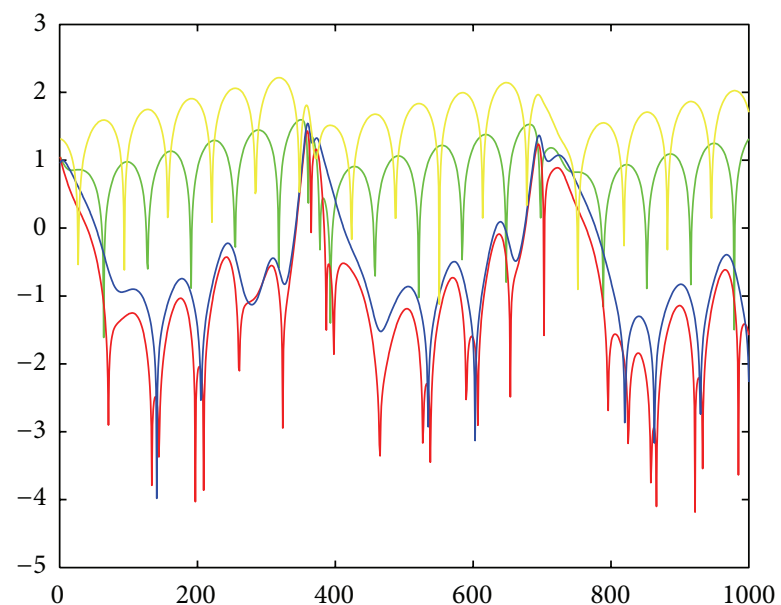

(d) The power spectrums of the dynamic system with $a=0.9, b=$ 20, $c=8, d=0.06$, and $e=25$

FIGURE 9: The power spectrums of the dynamic system when $x=11.28, y=-11.21, z=-9$, and $w=20.49$. (The red color stands for the power spectrum of $x$; the yellow color stands for the power spectrum of $y$; the blue color stands for the power spectrum of $z$; and the green color stands for the power spectrum of $w$.)

$$
\begin{gathered}
Z=z+\left(\frac{d}{2}+\frac{\sqrt{d^{2}+4 b c}}{2}\right), \\
W=w+b .
\end{gathered}
$$

Then, the dynamic system can be rewritten as follows:

$$
\begin{aligned}
\dot{X}= & a\left[X-\left(\frac{d}{2}+\frac{\sqrt{d^{2}+4 b c}}{2}\right)(2 a-b)\right. \\
& \left.-Y-\left(\frac{d}{2}+\frac{\sqrt{d^{2}+4 b c}}{2}\right)\right] \\
& -\left[Y+\left(\frac{d}{2}+\frac{\sqrt{d^{2}+4 b c}}{2}\right)\right] \\
& \times\left[Z-\left(\frac{d}{2}+\frac{\sqrt{d^{2}+4 b c}}{2}\right)\right]+(W-b),
\end{aligned}
$$

$$
\begin{aligned}
\dot{Y}= & -b\left[Y+\left(\frac{d}{2}+\frac{\sqrt{d^{2}+4 b c}}{2}\right)\right] \\
+ & {\left[X-\left(\frac{d}{2}+\frac{\sqrt{d^{2}+4 b c}}{2}\right)(2 a-b)\right] } \\
& \times\left[Z-\left(\frac{d}{2}+\frac{\sqrt{d^{2}+4 b c}}{2}\right)\right], \\
\dot{Z}= & -c\left[Z-\left(\frac{d}{2}+\frac{\sqrt{d^{2}+4 b c}}{2}\right)\right] \\
+ & d\left[X-\left(\frac{d}{2}+\frac{\sqrt{d^{2}+4 b c}}{2}\right)(2 a-b)\right] \\
+ & {\left[X-\left(\frac{d}{2}+\frac{\sqrt{d^{2}+4 b c}}{2}\right)(2 a-b)\right] }
\end{aligned}
$$




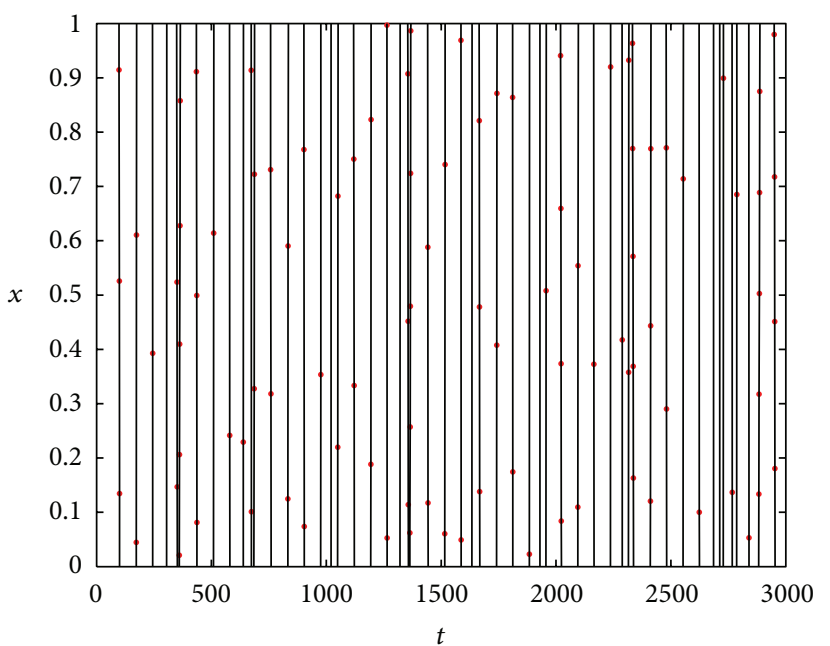

(a) The time chart for $x$

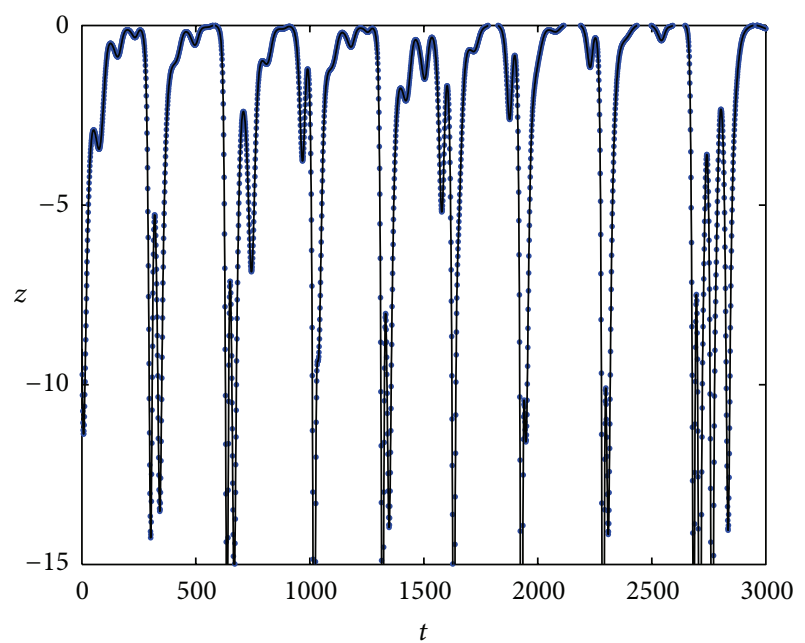

(c) The time chart for $z$

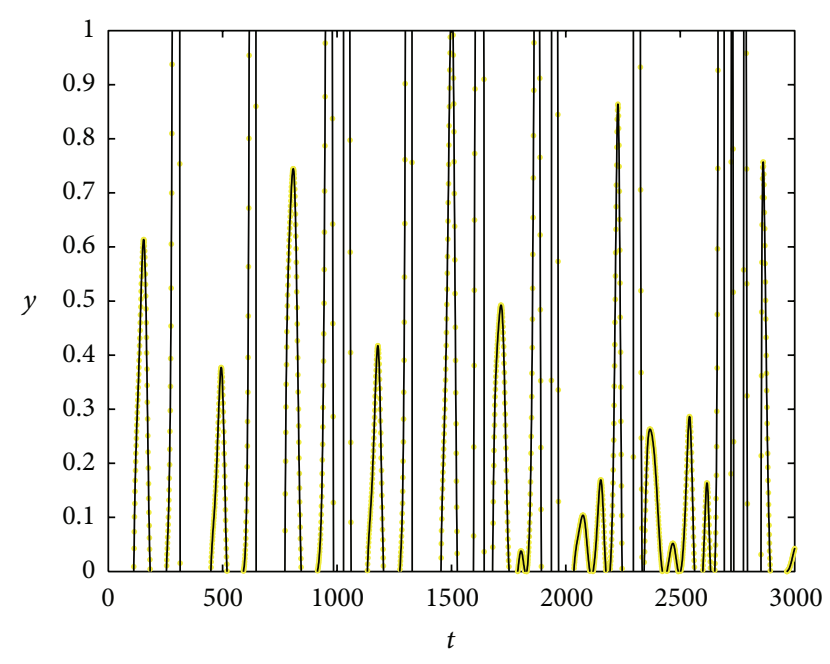

(b) The time chart for $y$

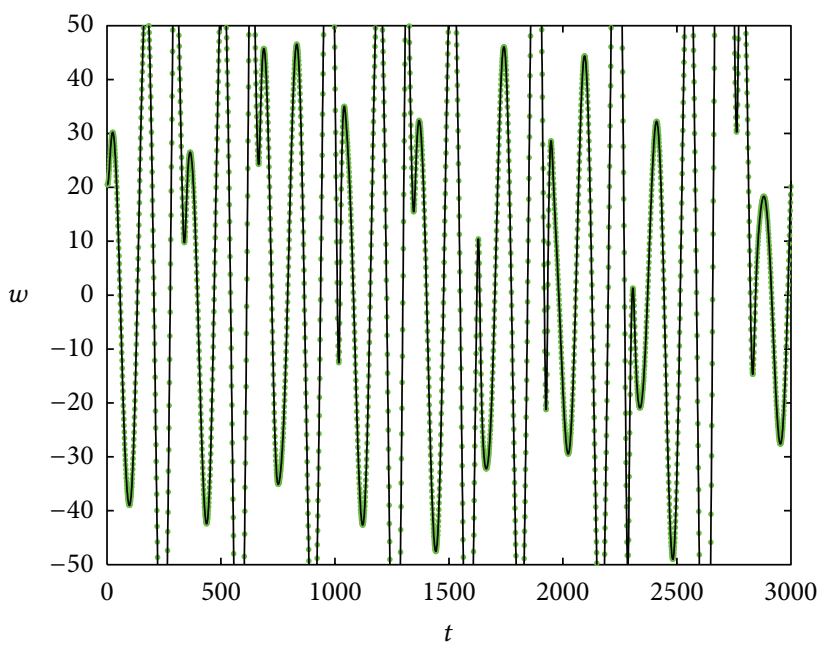

(d) The time chart for $w$

Figure 10: The time charts of the system with $x=11.28, y=-11.21, z=-9$, and $w=20.49$ and $a=0.98, b=12, c=6, d=0.06$, and $e=20$.

$$
\begin{gathered}
\times\left[Y+\left(\frac{d}{2}+\frac{\sqrt{d^{2}+4 b c}}{2}\right)\right] \\
\dot{W}=-e\left[X-\left(\frac{d}{2}+\frac{\sqrt{d^{2}+4 b c}}{2}\right)(2 a-b)\right] \\
+Y+\left(\frac{d}{2}+\frac{\sqrt{d^{2}+4 b c}}{2}\right) .
\end{gathered}
$$

We assume that the controlled dynamic system can be described as follows: assume that the controlled dynamic system can be described as follows:

$$
\begin{gathered}
\dot{X}=a\left[X-\left(\frac{d}{2}+\frac{\sqrt{d^{2}+4 b c}}{2}\right)(2 a-b)\right. \\
\left.-Y-\left(\frac{d}{2}+\frac{\sqrt{d^{2}+4 b c}}{2}\right)\right]
\end{gathered}
$$

$$
\begin{aligned}
- & {\left[Y+\left(\frac{d}{2}+\frac{\sqrt{d^{2}+4 b c}}{2}\right)\right] } \\
\times & {\left[Z-\left(\frac{d}{2}+\frac{\sqrt{d^{2}+4 b c}}{2}\right)\right]+(W-b)-k X, } \\
\dot{Y}= & -b\left[Y+\left(\frac{d}{2}+\frac{\sqrt{d^{2}+4 b c}}{2}\right)\right] \\
+ & {\left[X-\left(\frac{d}{2}+\frac{\sqrt{d^{2}+4 b c}}{2}\right)(2 a-b)\right] } \\
& \times\left[Z-\left(\frac{d}{2}+\frac{\sqrt{d^{2}+4 b c}}{2}\right)\right]-k Y, \\
\dot{Z}= & -c\left[Z-\left(\frac{d}{2}+\frac{\sqrt{d^{2}+4 b c}}{2}\right)\right]
\end{aligned}
$$




$$
\begin{aligned}
& +d\left[X-\left(\frac{d}{2}+\frac{\sqrt{d^{2}+4 b c}}{2}\right)(2 a-b)\right] \\
& +\left[X-\left(\frac{d}{2}+\frac{\sqrt{d^{2}+4 b c}}{2}\right)(2 a-b)\right] \\
& \times\left[Y+\left(\frac{d}{2}+\frac{\sqrt{d^{2}+4 b c}}{2}\right)\right]-k Z,
\end{aligned}
$$

$$
\begin{aligned}
\dot{W}= & -e\left[X-\left(\frac{d}{2}+\frac{\sqrt{d^{2}+4 b c}}{2}\right)(2 a-b)\right] \\
& +Y+\left(\frac{d}{2}+\frac{\sqrt{d^{2}+4 b c}}{2}\right)-k W .
\end{aligned}
$$

The Jacobian matrix of the controlled system is as follows:

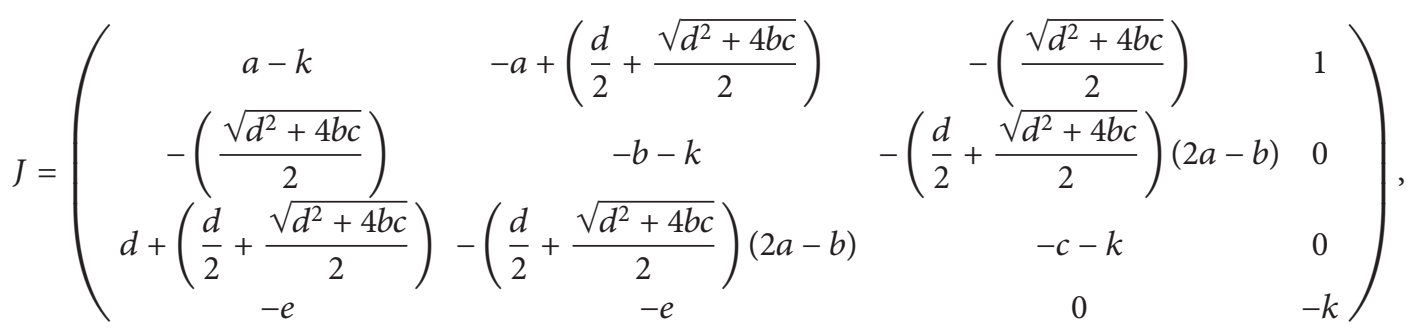

and the characteristic polynomial is:

$$
p(\lambda)=\lambda^{4}+m_{1} \lambda^{3}+m_{2} \lambda^{2}+m_{3} \lambda+m_{4},
$$

where

$$
\begin{aligned}
& m_{1}=b+c-4 k-1 ; \\
& m_{2}=\left(\sqrt{d^{2}+4 b c} / 2\right)-b-c+e-3 k+6 k^{2}-a^{2}\left(d^{2}+4 b c\right)-
\end{aligned}
$$

$\left(b^{2}\left(d^{2}+4 b c\right) / 4\right)+d \sqrt{d^{2}+4 b c}-a^{2} d^{2}-\left(b^{2} d^{2} / 4\right)+b c+3 b k+3 c k+$ $a b\left(d^{2}+4 b c\right)+a b d^{2}-2 a^{2} d \sqrt{d^{2}+4 b c}-\left(b^{2} d \sqrt{d^{2}+4 b c} / 2\right)+$ $2 a b d \sqrt{d^{2}+4 b c}$;

$$
m_{3}=-3 k^{2}+4 k^{3}+a^{2} d^{2}+\left(b^{2} d^{2} / 4\right)+\left(b\left(d^{2}+4 b c\right) / 4\right)+
$$
$\left(c\left(d^{2}+4 b c\right) / 4\right)+k\left(d^{2}+4 b c\right)-b c+b e+c e-2 b k-$ $2 c k+2 e k+a^{2}\left(d^{2}+4 b c\right)+\left(a \sqrt[3 / 2]{d^{2}+4 b c} / 2\right)+\left(b^{2}\left(d^{2}+\right.\right.$ $4 b c) / 4)-\left(b \sqrt[3 / 2]{d^{2}+4 b c} / 4\right)-\left(e \sqrt{d^{2}+4 b c} / 2\right)+\left(3 a d^{3} / 4\right)-$ $\left(3 b d^{3} / 8\right)+3 b k^{2}+3 c k^{2}+\left(3 b d \sqrt{d^{2}+4 b c} / 4\right)+\left(c d \sqrt{d^{2}+4 b c} / 4\right)-$ $2 a^{2} k\left(d^{2}+4 b c\right)-\left(b^{2} k\left(d^{2}+4 b c\right) / 2\right)+2 d k \sqrt{d^{2}+4 b c}-a b d^{2}+$ $\left(7 a d^{2} \sqrt{d^{2}+4 b c} / 4\right)+2 a^{2} d \sqrt{d^{2}+4 b c}-\left(7 b d^{2} \sqrt{d^{2}+4 b c} / 8\right)+$ $\left(b^{2} d \sqrt{d^{2}+4 b c} / 2\right)-2 a^{2} d^{2}-\left(b^{2} d^{2} k / 2\right)-a b\left(d^{2}+4 b c\right)+\left(3 a d\left(d^{2}+\right.\right.$ $4 b c) / 2)-\left(3 b d\left(d^{2}+4 b c\right) / 4\right)+2 b c k-4 a^{2} d k \sqrt{d^{2}+4 b c}-$ $b^{2} d k \sqrt{d^{2}+4 b c}+2 a b k\left(d^{2}+4 b c\right)-2 a b d \sqrt{d^{2}+4 b c}+2 a b d^{2} k+$ $4 a b d k \sqrt{d^{2}+4 b c}$;

$m_{4}=\left(\left(d^{2}+4 b c\right) k^{2} / 2\right)-b k^{2}+b k^{3}-c k^{2}+c k^{3}+e k^{2}-k^{3}+$ $k^{4}-a^{2} k^{2}\left(d^{2}+4 b c\right)-\left(b^{2} k^{2}\left(d^{2}+4 b c\right) / 4\right)+d k^{2} \sqrt{d^{2}+4 b c}-$ $a^{2} d^{2} e-\left(b^{2} d^{2} e / 4\right)+a^{2} d^{2} k+\left(b^{2} d^{2} k / 4\right)-\left(a e\left(d^{2}+4 b c\right) / 2\right)+$ $\left(b e\left(d^{2}+4 b c\right) / 4\right)+\left(b k\left(d^{2}+4 b c\right) / 4\right)+\left(c k\left(d^{2}+4 b c\right) / 4\right)+$ $b c e-b c k+b e k+c e k-a^{2} d^{2} k^{2}-\left(b^{2} d^{2} k^{2} / 4\right)-a^{2} e\left(d^{2}+\right.$ $4 b c)-\left(b^{2} e\left(d^{2}+4 b c\right) / 4\right)-\left(c e \sqrt{d^{2}+4 b c} / 2\right)+a^{2} k\left(d^{2}+4 b c\right)+$ $\left(a k \sqrt[3 / 2]{d^{2}+4 b c} / 2\right)+\left(b^{2} k\left(d^{2}+4 b c\right) / 4\right)-\left(b k \sqrt[3 / 2]{d^{2}+4 b c} / 4\right)-$ $\left(e k \sqrt{d^{2}+4 b c} / 2\right)-\left(3 a d^{2} e / 2\right)+\left(3 b d^{2} e / 4\right)+b c k^{4}+\left(3 a d^{3} k / 4\right)-$ $\left(3 b d^{3} k / 8\right)-2 a d e \sqrt{d^{2}+4 b c}+b d e \sqrt{d^{2}+4 b c}+a b k^{2}\left(d^{2}+4 b c\right)+$ $\left(3 b d k \sqrt{d^{2}+4 b c} / 4\right)+\left(c d k \sqrt{d^{2}+4 b c} / 4\right)+a b d^{2} e-a b d^{2} k-$ $2 a^{2} d e \sqrt{d^{2}+4 b c}-\left(b^{2} d e \sqrt{d^{2}+4 b c} / 2\right)\left(7 a d^{2} k \sqrt{d^{2}+4 b c} / 4\right)+$ $2 a^{2} d k \sqrt{d^{2}+4 b c}-\left(7 b d^{2} k \sqrt{d^{2}+4 b c} / 8\right)+\left(b^{2} d k \sqrt{d^{2}+4 b c} / 2\right)+$ $a b d^{2} k^{2}+a b e\left(d^{2}+4 b c\right)-a b k\left(d^{2}+4 b c\right)+\left(3 a d k\left(d^{2}+4 b c\right) / 2\right)-$ $\left(3 b d k\left(d^{2}+4 b c\right) / 4\right)-2 a^{2} d k^{2} \sqrt{d^{2}+4 b c}-\left(b^{2} d k^{2} \sqrt{d^{2}+4 b c} / 2\right)+$ $2 a b d e \sqrt{d^{2}+4 b c}-2 a b d k \sqrt{d^{2}+4 b c}+2 a b d k^{2} \sqrt{d^{2}+4 b c}$.
When

$$
a=1, \quad b=12, \quad c=5, \quad d=0.06, \quad e=0.1,
$$

the system is chaotic, and the conditions for the local stability of equilibrium are as follows:

$$
\begin{gathered}
H(1)=\left(m_{1}\right), \\
H(2)=\left(\begin{array}{cc}
m_{1} & 1 \\
m_{3} & m_{2}
\end{array}\right), \\
H(3)=\left(\begin{array}{ccc}
m_{1} & 1 & 0 \\
m_{3} & m_{2} & m_{1} \\
0 & m_{4} & m_{3}
\end{array}\right), \\
H(4)=\left(\begin{array}{cccc}
m_{1} & 1 & 0 & 0 \\
m_{3} & m_{2} & m_{1} & 1 \\
0 & m_{4} & m_{3} & m_{2} \\
0 & 0 & 0 & m_{4}
\end{array}\right) .
\end{gathered}
$$

For (20), they must satisfy $\operatorname{det} H_{j}>0(j=1,2,3,4)$, and then we get

$$
\begin{gathered}
\operatorname{det} H_{1}=m_{1}>0, \\
\operatorname{det} H_{2}=m_{1} m_{2}-m_{3}>0, \\
\operatorname{det} H_{3}=m_{1} m_{2} m_{3}-m_{3}^{2}-m_{1}^{2} m_{4}>0, \\
\operatorname{det} H_{4}=m_{4}\left(m_{1} m_{2} m_{3}-m_{3}^{2}-m_{1}^{2} m_{4}\right)>0 .
\end{gathered}
$$

And we can obtain the conditions for the local stability of equilibrium:

$$
\begin{gathered}
m_{1}>0, \\
m_{1} m_{2}-m_{3}>0, \\
m_{4}>0, \\
m_{1} m_{2} m_{3}>m_{3}^{2}+m_{1}^{2} m_{4},
\end{gathered}
$$


where

$$
\begin{gathered}
m_{1}=4 k+16+4 k+16 \\
m_{2}=6 k^{2}+48 k+104.032736 \\
m_{3}=4 k^{3}+48 k^{2}+208.065472 k+1980.465427712 \\
m_{4}=k^{4}+16 k^{3}+104.032736 k^{2} \\
+1980.465427712 k+9.934656
\end{gathered}
$$

Then, according to the condition of Routh-Hurwitz criteria, the local stability of equilibrium point E2 can be gained as follows:

$$
\begin{gathered}
4 k+16+4 k+16>0 \\
(4 k 16+4 k+16) \times\left(6 k^{2}+48 k+104.032736\right) \\
-\left(4 k^{3}+48 k^{2}+208.065472 k+1980.465427712\right)>0 \\
k^{4}+16 k^{3}+104.032736 k^{2}+1980.465427712 k \\
+9.934656>0 \\
(4 k+16+4 k+16) \times\left(6 k^{2}+48 k+104.032736\right) \\
\times\left(4 k^{3}+48 k^{2}+208.065472 k+1980.465427712\right) \\
>\left(4 k^{3}+48 k^{2}+208.065472 k+1980.465427712\right)^{2} \\
+(4 k+16+4 k+16)^{2} \\
\times\left(k^{4}+16 k^{3}+104.032736 k^{2}+1980.465427712 k\right. \\
\quad+9.934656) .
\end{gathered}
$$

When the control gain $k=80$ is chosen in the system, the corresponding four characteristic values are

$$
\begin{aligned}
\lambda_{1}=-1.6493, & \lambda_{2}=-0.1066, \\
\lambda_{3}=-0.8021+0.0022 i, & \lambda_{4}=-0.8021-0.0022 i .
\end{aligned}
$$

Obviously, the equilibrium point $E 2$ in chaotic state is driven into a steady state, and the goal of chaos control is realized.

Similarly, when

$$
\begin{gathered}
a=1, \quad b=12, \quad c=50, \\
d=0.06, \quad e=0.9,
\end{gathered}
$$

the system is in the hyperchaotic state. When $k=120$ is in the hyperchaotic system, the eigenvalues are

$$
\begin{array}{cc}
\lambda_{1}=-392.5879, & \lambda_{2}=94.9760, \\
\lambda_{3}=-123.1185, & \lambda_{4}=-120.2696 .
\end{array}
$$

Obviously $\lambda_{2}$ is the positive real number, and the rest three are negative real numbers. Thus, we can get the result that the hyperchaotic system undergoes a Hopf bifurcation at the equilibrium $E 2$.

When $k=216$, we get the eigenvalues according to the equilibrium point $E 2$ :

$$
\begin{gathered}
\lambda_{1}=-488.5879, \quad \lambda_{2}=-1.0240, \\
\lambda_{3}=-219.1185, \quad \lambda_{4}=-216.2696 .
\end{gathered}
$$

Here, $\lambda_{1}, \lambda_{2}, \lambda_{3}$, and $\lambda_{4}$ are negative real numbers, so the hyperchaotic system is restored into a steady state.

It is found that the controller gain $k$ can suppress the chaotic behavior to a steady state. Furthermore, hyperchaotic behavior can also be driven to a steady state or chaos or a periodic orbit because of a linear feedback control.

It draws conclusions that it shows a positive correlation between the degree of stability of the system and the value of the linear controller gain $k$. The larger the value of the linear controller gain $k$, the lower the degree of complexity of the system. It means that the new four-dimensional system tends to undergo towards the direction of a steady state. When the value of controller gain $k$ increases to a certain value, the hyperchaotic system reaches a steady state. The larger the value of controller gain $k$, the higher the performance of the stability of the system. Within certain parameters, $a=1$, $b=12, c=5, d=0.06$, and $e=0.1$, the system is chaotic, and the controller gain $k=80$ can drive the system to reach a steady state; when $a=1, b=12, c=50$, $d=0.06$, and $e=0.9$, the system is hyperchaotic, and at this time the controller gain $k=216$ can achieve the goal of making the original system to a steady state. The conclusion can be proved once again; the hyperchaos appears much more complex than chaos, and we need to increase the value of controller gain $k$ to achieve the goal of making the system in a steady state. For the other systems which generate chaos or hyperchaos, the hyperchaotic controller designed in this paper indicates that this is a simple and feasible way to control and adjust parameters when hyperchaotic or chaotic phenomenon happens in the dynamic system.

There is also a problem that we should pay attention to regarding chaos control, because the chosen control gain $k$ is difficult in the practice. Generally, we could analyze the equilibrium point and the stability of the chaotic or hyperchaotic system then map out the bifurcation diagram and calculate the Lyapunov exponents nearby the equilibrium point. At last, we get the coefficient control gain $k$. In this paper, we give Table 1 to express the values of control gain $k$ to help us select a suitable controlled value as fast as possible in practice. Table 1 is control tag.

Besides this, there are other methods for chaos control, such as frequency master method, the dynamics state feedback control, period-doubling bifurcation control, and neural network control, so we can choose proper control method according to our needs.

\section{Conclusion}

A system designed via state feedback control is proposed, and the corresponding complex dynamic characteristics are analyzed by exhibiting bifurcation diagrams, Lyapunov 
TABle 1: Control tag.

\begin{tabular}{|c|c|c|c|c|}
\hline Chaos: $k$ & 1 & 2 & 3 & 4 \\
\hline 20 & 0.4934 & -1.0493 & $-0.2021+0.0022 i$ & $-0.2021-0.0022 i$ \\
\hline 40 & 0.2934 & -1.2493 & $-0.4021+0.0022 i$ & $-0.4021-0.0022 i$ \\
\hline 60 & 0.0934 & -1.4493 & $-0.6021+0.0022 i$ & $-0.6021-0.0022 i$ \\
\hline 80 & -0.1066 & -1.6493 & $-0.8021+0.0022 i$ & $-0.8021-0.0022 i$ \\
\hline 100 & -0.3066 & -1.8493 & $-1.0021+0.0022 i$ & $-1.0021-0.0022 i$ \\
\hline 120 & -0.5066 & -2.0493 & $-1.2021+0.0022 i$ & $-1.2021-0.0022 i$ \\
\hline 140 & -0.7066 & -2.2493 & $-1.4021+0.0022 i$ & $-1.4021-0.0022 i$ \\
\hline 160 & -0.9066 & -2.4493 & $-1.6021+0.0022 i$ & $-1.6021-0.0022 i$ \\
\hline 180 & -1.1066 & -2.6493 & $-1.8021+0.0022 i$ & $-1.8021-0.0022 i$ \\
\hline 200 & -1.3066 & -2.8493 & $-2.0021+0.0022 i$ & $-2.0021-0.0022 i$ \\
\hline Hyperchaos & 1 & 2 & 3 & 4 \\
\hline 0.000001 & 214.9760 & -0.2696 & -3.1185 & -272.5879 \\
\hline 0.05 & 214.9260 & -0.3196 & -3.1685 & -272.6379 \\
\hline 0.5 & 214.4760 & -0.7696 & -3.6185 & -273.0879 \\
\hline 1 & 213.9760 & -1.2696 & -4.1185 & -273.5879 \\
\hline 2 & 212.9760 & -2.2696 & -5.1185 & -274.5879 \\
\hline 5 & 209.9760 & -5.2696 & -8.1185 & -277.5879 \\
\hline 10 & 204.9760 & -10.2696 & -13.1185 & -282.5879 \\
\hline 20 & 194.9760 & -20.2696 & -23.1185 & -292.5879 \\
\hline 40 & 174.9760 & -40.2696 & -43.1185 & -312.5879 \\
\hline 60 & 154.9760 & -60.2696 & -63.1185 & -332.5879 \\
\hline 80 & 134.9760 & -80.2696 & -83.1185 & -352.5879 \\
\hline 100 & 114.9760 & -100.2696 & -103.1185 & -372.5879 \\
\hline 120 & 94.9760 & -120.2696 & -123.1185 & -392.5879 \\
\hline 140 & 74.9760 & -140.2696 & -143.1185 & -412.5879 \\
\hline 160 & 54.9760 & -160.2696 & -163.1185 & -432.5879 \\
\hline 180 & 34.9760 & -180.2696 & -183.1185 & -452.5879 \\
\hline 200 & 14.9760 & -200.2696 & -203.1185 & -472.5879 \\
\hline 216 & -1.0240 & -216.2696 & -219.1185 & -488.5879 \\
\hline 220 & -5.0240 & -220.2696 & -223.1185 & -492.5879 \\
\hline 240 & -25.0240 & -240.2696 & -243.1185 & -512.5879 \\
\hline
\end{tabular}

exponents, Lyapunov dimensions, attractors, the power spectrums, the time chart, and so on. On the basis of the detailed numerical simulation as well as the theoretical analysis, it is observed that the system can generate both chaos and hyperchaos, and the behaviors of the hyperchaos are much more complex and abundant than the chaos.

A simple and effective controller which not only can drive the chaotic system to a steady state but also restore the hyperchaotic system to a periodic orbit or an equilibrium point is presented. A linear feedback controller is used to achieve a second control to the system based on the state feedback at the first time introduced and analyzed by Wang.

The next step of this paper is to further analyze and explore the effect of a second control, and there are several brave imaginations for the following analysis:

(1) what the difference between the first control and the second control is to the system in the aspects of effect of complexity and whether there is economic sense in practice;
(2) whether the goal will be realized for maximizing the benefits when the first control and the second control coordinate with and use the advantages of each other, complementary advantages. For example, whether the second controller can play a key role in the process of chaos control or hyperchaos control and realize the control for the second time.

\section{Acknowledgments}

The author extends this sincere thanks and appreciation to the institutions and people who have worked collaboratively to see that the aims and objectives to complete this research are attained. The author seizes this opportunity to thank the National Nature Science Foundation of China (Grant no. 61273231).

\section{References}

[1] P. C. Rech, "Chaos and hyperchao sina hopfield neural network," I Neurocomputing, vol. 74, pp. 3361-3364, 2011. 
[2] S. Y. Li, S. C. Huang, C. H. Yang, and Z. M. Ge, "Generating trichaos attractors with three positive Lyapunov exponents in new four order system via linear coupling," Nonlinear Dynamics, vol. 69, no. 3, pp. 805-816, 2012.

[3] K. P. Harikrishnan, R. Misra, and G. Ambika, "Revisiting the box counting algorithm for the correlation dimension analysis of hyperchaotic time series," Communications in Nonlinear Science and Numerical Simulation, vol. 17, no. 1, pp. 263-276, 2012.

[4] J. L. Kaplan and J. A. Yorke, "Chaotic behavior of multidimensional difference equations," in Functional Differential Equations and Approximation of Fixed Points, vol. 730 of Lecture Notes in Mathematics, pp. 204-227, Springer, Berlin, Germany, 1979.

[5] L. Feng and J. Yinlai, "Hopf bifurcation analysis and numerical simulation in a 4D-hyoerchaotic system," Nonlinear Dynamics, vol. 67, no. 4, pp. 2857-2864, 2012.

[6] E. N. Lörenz, "Deterministic nonperiodic flow," Journal of the Atmospheric Sciences, vol. 20, pp. 130-141, 1963.

[7] G. Chen and T. Ueta, "Yet another chaotic attractor," International Journal of Bifurcation and Chaos in Applied Sciences and Engineering, vol. 9, no. 7, pp. 1465-1466, 1999.

[8] J. L. Lü and G. Chen, "A new chaotic attractor coined," International Journal of Bifurcation and Chaos in Applied Sciences and Engineering, vol. 12, no. 3, pp. 659-661, 2002.

[9] L. Wang, "3-scroll and 4-scroll chaotic attractors generated from a new 3-D quadratic autonomous system," Nonlinear Dynamics, vol. 56, no. 4, pp. 453-462, 2009.

[10] L. Wang, "Yet another 3D quadratic autonomous system generating three-wing and four-wing chaotic attractors," Chaos, vol. 19, no. 1, Article ID 013107, 6 pages, 2009.

[11] J. H. Ma and Q. Gao, "Stability and Hopf bifurcations in a business cycle model with delay," Applied Mathematics and Computation, vol. 215, no. 2, pp. 829-834, 2009.

[12] J. H. Ma and Q. Gao, "Analysis on the chaotic motion of a stochastic nonlinear dynamic system," International Journal of Computer Mathematics, vol. 87, no. 14, pp. 3266-3272, 2010.

[13] B. G. Xin, J. H. Ma, and Q. Gao, "Complex dynamics of an adnascent-type game model," Discrete Dynamics in Nature and Society, vol. 2008, Article ID 467972, 12 pages, 2008.

[14] C. C. Yang, "One input control of exponential synchronization for a four-dimensional chaotic system," Applied Mathematics and Computation, vol. 219, no. 10, pp. 5152-5161, 2013.

[15] X. Wang, B. Xu, and G. Luo, "Anasynchronous communication system based on the hyperchaotic system of 6th-order cellular neuralnetwork," Optics Communications, vol. 285, pp. 54015405, 2012.

[16] M. J. Correia and P. C. Rech, "Hyperchaotic states in the parameter-space," Applied Mathematics and Computation, vol. 218, no. 12, pp. 6711-6715, 2012.

[17] S. R. Choudhury and R. A. van Gorder, "Competitive modes as reliable predictors of chaos versus hyperchaos and as geometric mappings accurately delimiting attractors," Nonlinear Dynamics, vol. 69, no. 4, pp. 2255-2267, 2012.

[18] S. Yu and G. R. Chen, "Anti-control of continuous-time dynamical systems," Communications in Nonlinear Science and Numerical Simulation, vol. 17, no. 6, pp. 2617-2627, 2012.

[19] P. Yu and M. Han, "Bifurcation of limit cycles in 3rd-order Z2 Hamiltonian planar vector fields with 3rd-order perturbations," Communications in Nonlinear Science and Numerical Simulation, vol. 18, no. 4, pp. 978-988, 2013.
[20] K. Sun, X. Liu, C. Zhu, and J. C. Sprott, "Hyperchaos and hyperchaos control of the sinusoidally forced simplified Lorenz system," Nonlinear Dynamics, vol. 69, no. 3, pp. 1383-1391, 2012.

[21] H. Yu, G. Cai, and Y. Li, "Dynamic analysis and control of a new hyperchaotic finance system," Nonlinear Dynamics, vol. 67, no. 3, pp. 2171-2182, 2012.

[22] W. Wu, Z. Chen, and Z. Yuan, "The evolution of a novel fourdimensional autonomous system: among 3-torus, limit cycle, 2torus, chaos and hyperchaos," Chaos, Solitons and Fractals, vol. 39, pp. 2340-2356, 2009.

[23] Z. Wang, S. Cang, E. O. Ochola, and Y. Sun, "A hyperchaotic system without equilibrium," Nonlinear Dynamics, vol. 69, no. 1-2, pp. 531-537, 2012.

[24] M. Sekikawa, N. Inaba, T. Tsubouchi, and K. Aihara, "Novel bifurcation structure generated in piecewise-linear three-LC resonant circuit and its Lyapunov analysis," Physica D, vol. 241, pp. 1169-1178, 2012. 


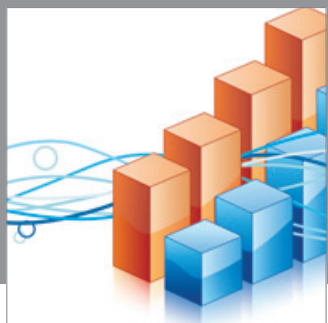

Advances in

Operations Research

mansans

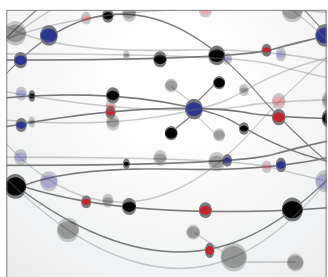

The Scientific World Journal
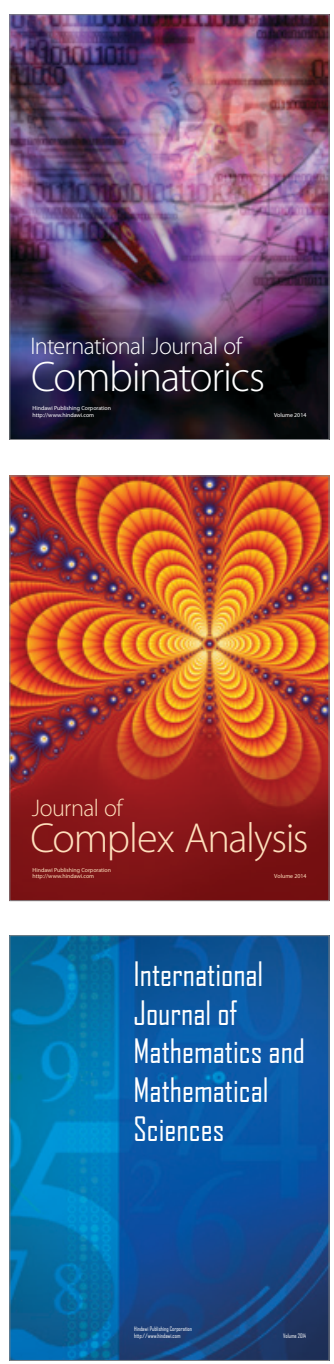
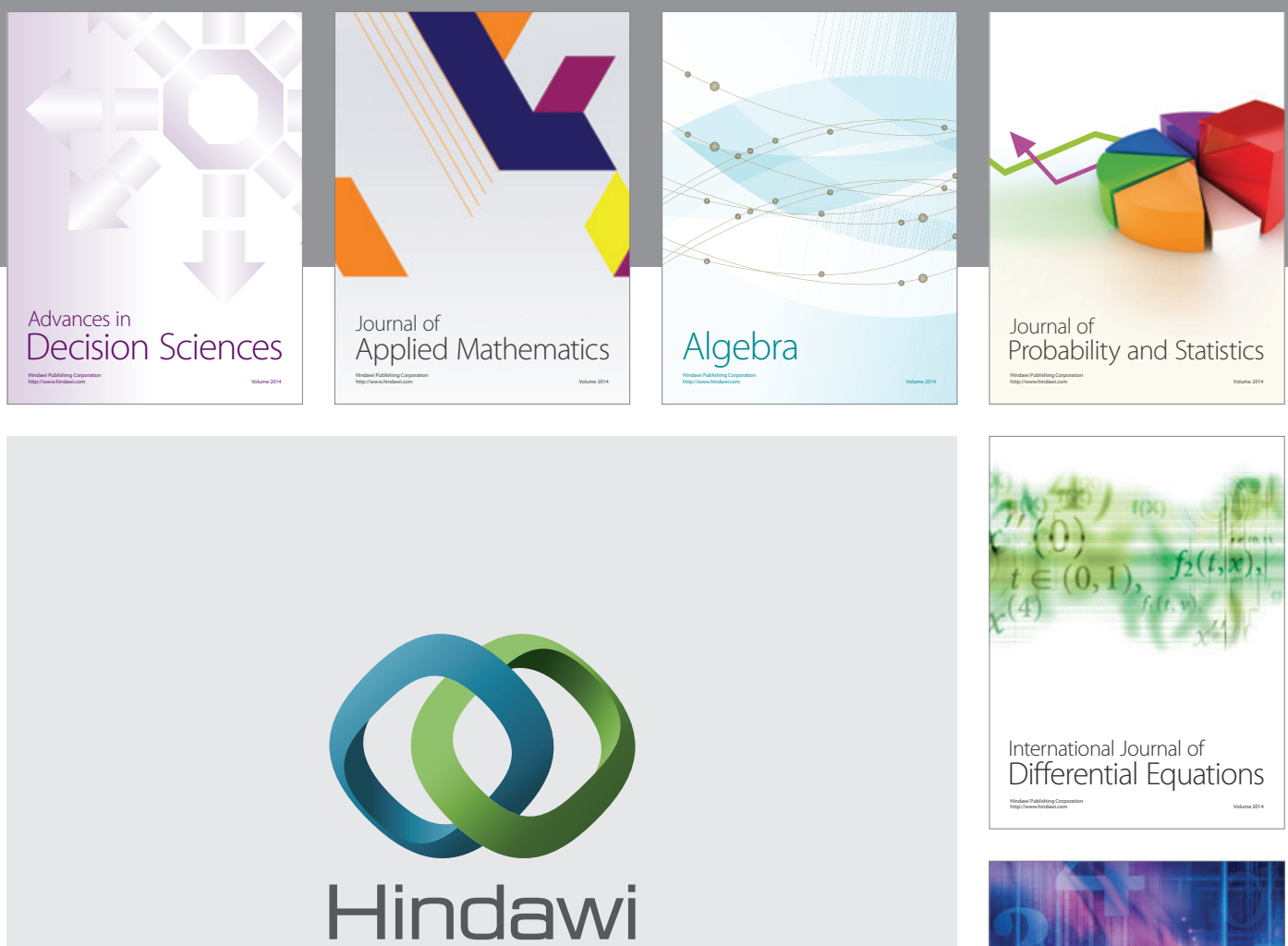

Submit your manuscripts at http://www.hindawi.com
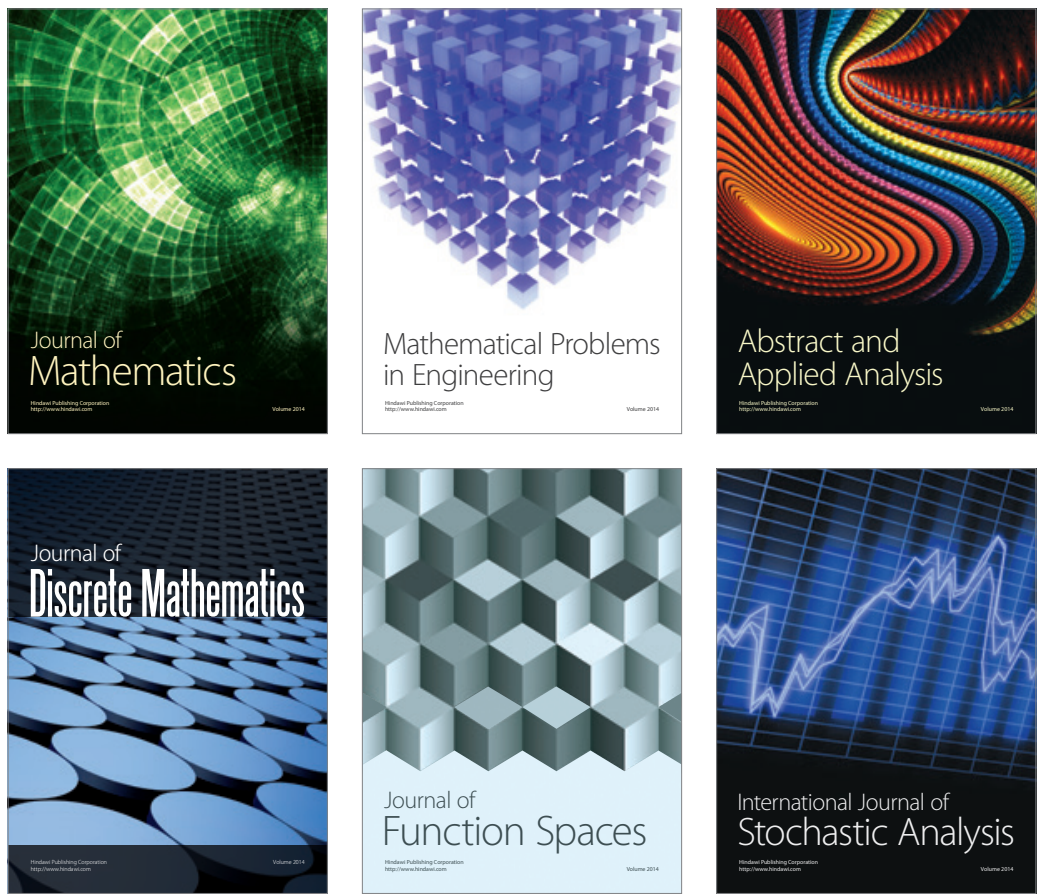

Journal of

Function Spaces

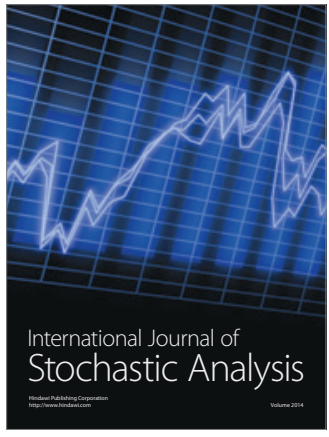

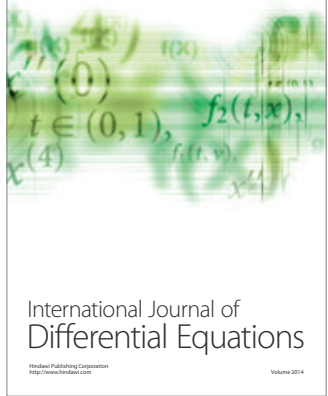
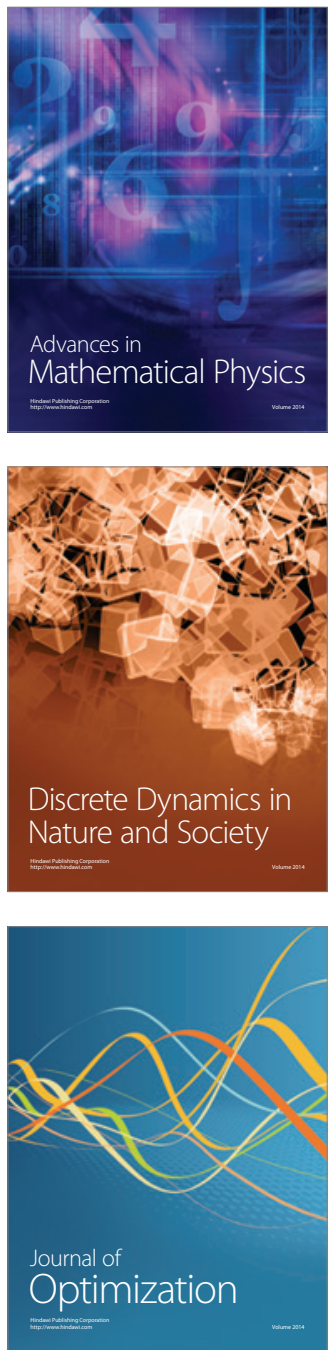